九州大学学術情報リポジトリ

Kyushu University Institutional Repository

\title{
An iterative method for obtaining a nonlinear solution for the temperature distribution of a spinning spherical body irradiated by a central star
}

Sekiya, Minoru

Department of Earth and Planetary Sciences, Faculty of Sciences, Kyushu University

Shimoda, Akihito A.

Department of Earth and Planetary Sciences, Faculty of Sciences, Kyushu University

Wakita, Shigeru

Department of Earth and Planetary Sciences, Faculty of Sciences, Kyushu University

http://hdl. hand le. net/2324/27273

出版情報: Planetary and Space Science. 60 (1)，pp.304-313，2012-01. Elsevier バージョン：

権利関係: (C) 2011 Elsevier Ltd. 


\title{
An iterative method for obtaining a nonlinear solution for the temperature distribution of a spinning spherical body irradiated by a central star
}

\author{
M. Sekiya*, A. A. Shimoda, S. Wakita \\ Department of Earth and Planetary Sciences, Faculty of Sciences, 33 Kyushu University, \\ Hakozaki, Fukuoka 812-8581, Japan
}

\begin{abstract}
We developed an iterative method for determining the three-dimensional temperature distribution in a spherical spinning body that is irradiated by a central star. The seasonal temperature change due to the orbital motion is ignored. It is assumed that material parameters such as the thermal conductivity and the thermometric conductivity are constant throughout the spherical body. A general solution for the temperature distribution inside a body is obtained using spherical harmonics and spherical Bessel functions. The surface boundary condition contains a term obtained using the StefanBoltzmann law and is nonlinear with respect to temperature because it is dependent on the fourth power of temperature. The coefficients of the general solution are fitted to satisfy the surface boundary condition by using the iterative method. We obtained solutions that satisfy the nonlinear boundary condition within $0.1 \%$ accuracy. We calculated the rate of change in
\end{abstract}

\footnotetext{
*Corresponding author. Phone: +81 92642 2669. Fax: +81 926422684.

Email addresses: sekiya.minoru.393@m.kyushu-u.ac.jp (M. Sekiya), shimoda.akihito@geo.kyushu-u.ac.jp (A. A. Shimoda), shigeru@geo.kyushu-u.ac.jp (S. Wakita) 
the semimajor axis due to the diurnal Yarkovsky effect using the linear and nonlinear solutions. The maximum difference between the rates calculated using the two sets of solutions is $13 \%$. Therefore current understanding of the diurnal Yarkovsky effect based on linear solutions is fairly good.

Keywords: Temperature, radiation, asteroids, small bodies, meteorites

\section{Introduction}

In planetary sciences, accurate determination of the temperature distribution in a small body that is irradiated by a central star is important. For example, the radiation reaction force acting on a spinning and revolving body causes the time evolution of the orbital elements; this is known as the Yarkovsky effect (Bottke et al., 2006). Models of the surface temperature are also important in analyzing the observed thermal emission of asteroids and asteroid-like objects.

In most calculations of the Yarkovsky effect, the temperature distributions in small bodies were determined by linear approximation of the Stefan-Boltzmann law (see Bottke et al. (2006) and the references therein). The temperature $T$ is expressed as the sum of the global average temperature $T_{a v}$ and the difference between the temperature and the global average temperature $\Delta T$. The fourth power of the temperature is expanded as $T^{4}=T_{a v}^{4}+4 T_{a v}^{3} \Delta T+6 T_{a v}^{2} \Delta T^{2}+4 T_{a v} \Delta T^{3}+\Delta T^{4}$, and the second- and higherorder terms of $\Delta T$ are omitted; a temperature distribution determined by using this approximation is called a linear temperature distribution or a solution. On the other hand, a temperature distribution determined by including the effects of the nonlinear terms of $\Delta T$ is called a nonlinear temperature 
distribution or a nonlinear solution.

Several authors have developed methods for determining the nonlinear temperature distributions. Some examples of studies in which the temperature distributions were determined by using the nonlinear boundary condition and by employing analytical or semi-analytical theories are as follows: Peterson (1976) developed a second-order theory in $\Delta T$. Vokrouhlický and Farinella (1999) developed a semianalytical-nonlinear theory of the seasonal Yarkovsky effect. Breiter et al. (2010) developed a semianalytical method based on plane-parallel geometry for a body with an arbitrary shape; nonlinear boundary conditions are handled by using an iterative, fast Fouriertransform-based solver. There are also a lot of numerical works which include the nonlinearity of thermal emission, 1D thermal conduction and the beaming (i.e., excess emission to the solar direction compared to the Lambertian surface) due to the surface roughness (e.g., Lagerros (1998); Spencer (1990); Spencer et al. (1989)).

In this paper, we developed a simple iterative method for determining the temperature distribution of a spinning spherical body that is irradiated by a central star. The seasonal temperature change due to the orbital motion is ignored. A body is assumed to be homogeneous, and it is assumed that thermal conductivity, thermometric conductivity, and surface absorption and emission coefficients of the body remain constant. Using the method developed by Vokrouhlický (1998), a general solution of the temperature distribution is obtained analytically. The arbitrary constants of the general solution should be determined by fitting the surface boundary condition, which is expressed by the equilibrium of heating by the central star, cooling due to the thermal 
radiation and the thermal-conduction cooling (or heating) at the surface. The cooling due to the thermal radiation is expressed by the Stefan-Boltzmann law according to which the relationship between the energy flux radiated and temperature is nonlinear. Vokrouhlický (1998) performed the fitting by the linear approximation. On the other hand, the method developed in this paper can be used to obtain the arbitrary constants of the general solution that satisfies the nonlinear boundary condition with an arbitrary accuracy.

The results show that the maximum and minimum temperatures reached in this study are significantly different from those in the case of the linear solution for a large body. The maximum difference between the rates of change of the semimajor axis due to the diurnal Yarkovsky effect determined based on the linear and nonlinear solutions is $13 \%$. Therefore current understanding of the diurnal Yarkovsky effect based on linear solutions is fairly good. In this sense, the results of our calculation may not be so important for the diurnal Yarkovsky effect. However, the method developed in this paper would be applicable to any thermal problem concerning spherical bodies that are irradiated from the outside and cool by thermal radiation. The subtle surface-temperature difference of asteroids would be important for observational astronomers.

\section{Basic equations}

We consider a spherical body that spins at a constant angular velocity $\tilde{\omega}=$ $2 \pi / \tilde{P}$, where $\tilde{P}$ is the spin period (hereafter, we use a tilde for dimensional variables in order to distinguish them from the corresponding dimensionless variables that will be introduced later). The orbital motion of the body is 
neglected, and the body is assumed to be irradiated by a parallel beam of light traveling in a constant direction from a central star. In other words, we neglect any seasonal effect. The luminosity of the central star, $\tilde{L}_{*}$, and the orbital radius of the body, $\tilde{a}$, are assumed to be constant; therefore, the stellar flux $\tilde{F}_{*}=\tilde{L}_{*} /\left(4 \pi \tilde{a}^{2}\right)$ will also be constant. All relevant physical properties of a body, such as the absorption constant $\alpha$ and the emissivity $\epsilon$ of the surface, thermal conductivity $\tilde{k}_{T}$, and the thermometric conductivity $\tilde{\kappa}=\tilde{k}_{T} /(\tilde{\rho} \tilde{C})$, where $\tilde{\rho}$ is the material density and $\tilde{C}$ is the specific heat, are assumed to be constant throughout the object and to remain constant with time.

\section{Figure 1}

We use a coordinate system $(\tilde{x}, \tilde{y}, \tilde{z})$ with the origin at the center of the body; the body spins around the $\tilde{z}$ axis, and the beam of the light from the central star travels in the $\tilde{x}-\tilde{z}$ plane at an angle $\theta_{*}$ from the $\tilde{z}$ axis, as shown in Fig. 1. The thermal conduction equation is expressed as follows:

$$
\frac{\partial \tilde{T}}{\partial \tilde{t}}+\tilde{\mathrm{v}} \cdot \nabla \tilde{T}=\tilde{\kappa} \nabla^{2} \tilde{T}
$$

where $\tilde{T}$ is the temperature, and the second term on the left-hand side (LHS) represents the advection of the temperature with a velocity $\tilde{\mathrm{v}}$ (e.g., Eq. (50.2) of Landau and Lifshitz (1987)). If one uses a coordinate system that moves with the body, the advection term will be equal to zero. However, in our system, the body moves because it is spinning, and therefore the second term remains. We ignore the revolutional motion around the central star, and take only the spin motion of the body into account; we assume that the 
temperature distribution is steady, and therefore, the first term in Eq. (1) will be equal to zero.

Now we use the spherical coordinates $(\tilde{r}, \theta, \phi)$ shown in Fig. 1, where $\tilde{r}$ is the radial coordinate $(\tilde{r}=\tilde{R}$ on the surface of a body). The thermalconduction equation for a steady temperature distribution is as follows:

$$
\tilde{\omega} \frac{\partial \tilde{T}}{\partial \phi}=\frac{\tilde{\kappa}}{\tilde{r}^{2}}\left\{\frac{\partial}{\partial \tilde{r}}\left(\tilde{r}^{2} \frac{\partial \tilde{T}}{\partial \tilde{r}}\right)+\left[\frac{1}{\sin \theta} \frac{\partial}{\partial \theta}\left(\sin \theta \frac{\partial \tilde{T}}{\partial \theta}\right)+\frac{1}{\sin ^{2} \theta} \frac{\partial^{2} \tilde{T}}{\partial \phi^{2}}\right]\right\},
$$

where the term on the LHS represents the advection due to the body's spin.

The absorbed energy of the stellar radiation per unit time and per unit surface area of the spherical body, which we call stellar heating rate in the following, is given by

$$
\tilde{\Gamma}(\cos \Theta)= \begin{cases}\alpha \tilde{F}_{*} \cos \Theta & \text { for } \quad 0 \leq \Theta<\pi / 2 \\ 0 & \text { for } \pi / 2 \leq \Theta \leq \pi\end{cases}
$$

where $\Theta$ is the angle between the direction of the central star and of a point with the spherical coordinates $(\tilde{R}, \theta, \phi)$ on the surface of the body when viewed from the center of the body, as shown in Fig. 1.

The surface boundary condition is expressed as the energy balance of the radiative cooling, thermal conduction, and heating by the central star per unit time and per unit surface area of a body as follows:

$$
\left[\epsilon \tilde{\sigma} \tilde{T}^{4}+\tilde{k}_{T} \frac{\partial \tilde{T}}{\partial \tilde{r}}\right]_{\tilde{r}=\tilde{R}}=\tilde{\Gamma}(\cos \Theta)
$$

where $\tilde{\sigma}$ is the Stefan-Boltzmann constant.

The total balance of the stellar heating rate and radiative cooling rate is expressed as:

$$
\pi \tilde{R}^{2} \alpha \tilde{F}_{*}=4 \pi \tilde{R}^{2} \epsilon \tilde{\sigma}<<\tilde{T}^{4}>_{\phi}>_{\theta}
$$


where $<>_{\phi} \equiv[1 /(2 \pi)] \int_{-\pi}^{\pi} d \phi$ is the mean with respect to the longitude, and $<>_{\theta} \equiv(1 / 2) \int_{-1}^{1} d \cos \theta$ is the mean with respect to the colatitude. Note that the global average of the heat conduction flux at the surface must be zero for a steady state. We define the global average temperature as $\tilde{T}_{a v} \equiv$ $\left[<<\tilde{T}^{4}>_{\phi}>_{\theta}\right]^{1 / 4}=\left[\alpha \tilde{F}_{*} /(4 \epsilon \tilde{\sigma})\right]^{1 / 4}=280\left[(\alpha / \epsilon)\left(\tilde{L}_{*} / \tilde{L}_{\odot}\right)\right]^{1 / 4}(\tilde{a} / 1 \mathrm{AU})^{-1 / 2}[\mathrm{~K}]$, where $\tilde{L}_{\odot}$ is the solar luminosity.

In the following part of the paper, we use the dimensionless temperature $T \equiv \tilde{T} / \tilde{T}_{a v}$, radius $r \equiv \tilde{r} / \tilde{R}$, and angular velocity $\omega \equiv \tilde{\omega} \tilde{R}^{2} / \tilde{\kappa}$. The global average temperature can be considered to be $T_{a v}=1$. Using these variables, the thermal conduction equation (Eq. (2)) is expressed as

$$
\omega \frac{\partial T}{\partial \phi}=\frac{1}{r^{2}}\left\{\frac{\partial}{\partial r}\left(r^{2} \frac{\partial T}{\partial r}\right)+\left[\frac{1}{\sin \theta} \frac{\partial}{\partial \theta}\left(\sin \theta \frac{\partial T}{\partial \theta}\right)+\frac{1}{\sin ^{2} \theta} \frac{\partial^{2} T}{\partial \phi^{2}}\right]\right\} .
$$

By dividing Eq. (4) by $4 \epsilon \tilde{\sigma} \tilde{T}_{a v}^{4}=\alpha \tilde{F}_{*}$, we get a dimensionless form of the boundary condition:

$$
\left[\frac{1}{4} T^{4}+q_{\text {global }} \frac{\partial T}{\partial r}\right]_{r=1}=\Gamma(\cos \Theta)
$$

where

$$
\Gamma(\cos \Theta) \equiv \frac{\tilde{\Gamma}(\cos \Theta)}{\alpha \tilde{F}_{*}}= \begin{cases}\cos \Theta & \text { for } \quad 0 \leq \Theta<\pi / 2 \\ 0 & \text { for } \pi / 2 \leq \Theta \leq \pi\end{cases}
$$

is the dimensionless form of the stellar heating rate, and

$$
q_{\text {global }} \equiv \frac{\tilde{k}_{T} \tilde{T}_{a v} / \tilde{R}}{\alpha \tilde{F}_{*}}
$$

is the dimensionless parameter that is a characteristic value of the global thermal conduction flux for the temperature gradient $\tilde{T}_{a v} / \tilde{R}$ normalized by the stellar heating rate $\alpha \tilde{F}_{*}$. Note that $q_{\text {global }}$ was denoted as $\lambda$ in Vokrouhlický (1998). 
Now, we develop an iterative method for determining the temperature distribution that satisfies Eqs. (6) and (7). We choose $T^{(0)}=1$ as the zeroth order solution that satisfies Eq. (6). The $j$-th order solution is expressed as follows:

$$
T^{(j)}=T^{(j-1)}+\delta T^{(j)}
$$

where $\delta T^{(j)}$ is the difference between the $j$-th order and the $(j-1)$-th order solutions. We expect that $\left|\delta T^{(j)}\right| /\left|\delta T^{(j-1)}\right|=O(\varepsilon)$, where $\varepsilon$ is a positive number smaller than unity. Then, $\left|\delta T^{(j)}\right|=O\left(\varepsilon^{j}\right)$ and the solution converges as $j \rightarrow \infty$.

Because Eq. (6) is linear in $T$, its difference $\delta T^{(j)}$ too must satisfy Eq. (6). In order to solve Eq. (6) for $\delta T^{(j)}$, we expand $\delta T^{(j)}$ using spherical harmonics

$$
\delta T^{(j)}=\sum_{n=0}^{\infty} \sum_{m=0}^{n} \delta T_{n m}^{(j)}
$$

where

$$
\delta T_{n m}^{(j)}=\delta C_{n m}^{(j)}(r) P_{n}^{m}(\cos \theta) \cos (m \phi)+\delta S_{n m}^{(j)}(r) P_{n}^{m}(\cos \theta) \sin (m \phi) .
$$

Here, $P_{n}^{m}(\cos \theta)$ is the associated Legendre function of the first kind, and $\delta C_{n m}^{(j)}(r)$ and $\delta S_{n m}^{(j)}(r)$ are real functions of $r$, which will be determined as shown below. Note that the spherical harmonics, $P_{n}^{m}(\cos \theta) \cos (m \phi)$ with $0 \leq n$ and $0 \leq m \leq n$ and $P_{n}^{m}(\cos \theta) \sin (m \phi)$ with $1 \leq n$ and $1 \leq m \leq n$ form a complete orthogonal system for a continuous real function of $(\theta, \phi)$ (we do not need terms with $m=0$ for $P_{n}^{m}(\cos \theta) \sin (m \phi)$, because $\left.\sin (m \phi)=0\right)$. Because the thermal conduction equation is linear, we can solve each term of the spherical harmonics separately. By substituting Eq. (12) into Eq. (6) 
and comparing each term proportional to $\cos (m \phi)$ and $\sin (m \phi)$, we get

$$
\frac{d}{d r}\left[r^{2} \frac{d}{d r} \delta C_{n m}^{(j)}(r)\right]-n(n+1) \delta C_{n m}^{(j)}(r)-m \omega r^{2} \delta S_{n m}^{(j)}(r)=0
$$

and

$$
\frac{d}{d r}\left[r^{2} \frac{d}{d r} \delta S_{n m}^{(j)}(r)\right]-n(n+1) \delta S_{n m}^{(j)}(r)+m \omega r^{2} \delta C_{n m}^{(j)}(r)=0
$$

When $m=0$, the solution that is regular at $r=0$ is

$$
\delta C_{n 0}^{(j)}(r)=\delta C_{n}^{(j)} r^{n}
$$

where $\delta C_{n}^{(j)}$ is a constant real number that can be determined on the basis of the surface boundary condition. When $m>0$, it is convenient to define a complex variable $\delta E_{n m}^{(j)}(r) \equiv \delta C_{n m}^{(j)}(r)-i \delta S_{n m}^{(j)}(r)$, where $i$ is the imaginary unit. Equations (13) and (14) can be merged into one equation,

$$
\frac{d}{d r}\left[r^{2} \frac{d}{d r} \delta E_{n m}^{(j)}(r)\right]-n(n+1) \delta E_{n m}^{(j)}(r)-i m \omega r^{2} \delta E_{n m}^{(j)}(r)=0 .
$$

A solution of this equation, which is regular at $r=0$, is $j_{n}\left(\beta_{m} r\right)$, where $j_{n}$ is the spherical Bessel function of the first kind and $\beta_{m} \equiv(-i m \omega)^{1 / 2}$ (Vokrouhlický, 1998). Because $j_{n}\left(\beta_{m} r\right)$ and its derivative $\beta_{m} j_{n}^{\prime}\left(\beta_{m} r\right)$ may take very large or very small values, we multiply them by $B_{n}\left(\beta_{m}\right)$, a coefficient that is dependent on $n$ and $\beta_{m}$, as follows:

$$
\hat{j}_{n, m}\left(\beta_{m} r\right) \equiv B_{n}\left(\beta_{m}\right) j_{n}\left(\beta_{m} r\right)
$$

and

$$
\hat{j}_{n, m}^{\prime}\left(\beta_{m} r\right) \equiv B_{n}\left(\beta_{m}\right) \beta_{m} j_{n}^{\prime}\left(\beta_{m} r\right) .
$$

Thus, we get appropriate values of $\hat{j}_{n, m}\left(\beta_{m} r\right)$ and $\hat{j}_{n, m}^{\prime}\left(\beta_{m} r\right)$ for computation. The detailed procedures for determining these coefficients and for calculating 
these functions are given in Appendix A. We obtain a general solution that is regular at $r=0$,

$$
\delta E_{n m}^{(j)}(r)=\delta \mathcal{E}_{n m}^{(j)} \hat{j}_{n, m}\left(\beta_{m} r\right)
$$

where $\delta \mathcal{E}_{n m}^{(j)}$ is a constant complex number, and it will be determined on the basis of the surface boundary condition. Thus, we get general solutions of the thermal conduction equation, which are

$$
\delta T_{n 0}^{(j)}(r, \theta)=\delta C_{n}^{(j)} r^{n} P_{n}(\cos \theta)
$$

and

$$
\delta T_{n m}^{(j)}(r, \theta, \phi)=\Re\left[\delta \mathcal{E}_{n m}^{(j)} \hat{j}_{n, m}\left(\beta_{m} r\right) \exp (i m \phi)\right] P_{n}^{m}(\cos \theta),
$$

where $\Re$ represents the real part. Equation (21) is used when $0<m \leq n$.

Substituting Eq. (10) into Eq. (7), we get

$$
\left[\frac{1}{4}\left(T^{(j-1)}+\delta T^{(j)}\right)^{4}+q_{g l o b a l}\left(\frac{\partial T^{(j-1)}}{\partial r}+\frac{\partial \delta T^{(j)}}{\partial r}\right)\right]_{r=1}=\Gamma(\cos \Theta) .
$$

By expanding the nonlinear term using $\delta T^{(j)}$ and omitting the terms on the order of $o\left(\varepsilon^{j}\right)$, we get $\left(T^{(j-1)}+\delta T^{(j)}\right)^{4} \approx\left(T^{(j-1)}\right)^{4}+4 \delta T^{(j)}$. Thus, we get the following boundary condition:

$$
\left[\delta T^{(j)}+q_{\text {global }} \frac{\partial \delta T^{(j)}}{\partial r}\right]_{r=1}=\Gamma(\cos \Theta)-\Lambda^{(j-1)}(\theta, \phi)-q_{\text {global }}\left[\frac{\partial T^{(j-1)}}{\partial r}\right]_{r=1} .
$$

where we denote the dimensionless radiative-cooling-rate by $\Lambda^{(j-1)}(\theta, \phi) \equiv$ $\left[\frac{1}{4}\left(T^{(j-1)}\right)^{4}\right]_{r=1}$.

Because we have already obtained the general solutions of the thermal conduction equation for $\delta T^{(j)}$ by performing the expansion on the basis of spherical harmonics and because Eq. (23) is linear in $\delta T^{(j)}$, we can get a 
solution that satisfies Eq. (23) by expanding the right-hand side (RHS) of Eq. (23) on the basis of spherical harmonics. The last term on the RHS of Eq. (23) has been already obtained when we are going to calculate $\delta T^{(j)}$ by employing the iterative method.

\section{Figures 2 and 3}

The first term on the RHS of Eq. (23) is written as

$$
\Gamma(\cos \Theta)=\sum_{n=0}^{\infty} \Gamma_{n} P_{n}(\cos \Theta),
$$

where each coefficient $\Gamma_{n}$ is given by

$$
\begin{aligned}
\Gamma_{n} & =\frac{2 n+1}{2} \int_{-1}^{1} \Gamma(\cos \Theta) P_{n}(\cos \Theta) d \cos \Theta \\
& =\frac{2 n+1}{2} \int_{0}^{1} x P_{n}(x) d x .
\end{aligned}
$$

The first three coefficients are $\Gamma_{0}=1 / 4, \Gamma_{1}=1 / 2$, and $\Gamma_{2}=5 / 16$. We have $\Gamma_{n}=0$ for odd values of $n \geq 3$, and

$$
\Gamma_{n}=\frac{2 n+1}{2} \frac{(-1)^{(n / 2)-1}(n-3) ! !}{(n+2) ! !}
$$

for even values of $n \geq 4$ (e.g., Eq. (8.14.15) of Abramowitz and Stegun (1964)). It is convenient to use the recurrence formula,

$$
\Gamma_{n}=-\left(\frac{2 n+1}{2 n-3}\right)\left(\frac{n-3}{n+2}\right) \Gamma_{n-2}
$$

to perform the numerical calculation. We approximate the sum over $0 \leq$ $n \leq \infty$ in Eq. (24) by replacing $\infty$ with a large number $n_{\max }$. As expected from Eq. (27), $\Gamma_{n}$ converges very slowly. However, $n_{\max }=64$ provides a 
sufficiently good approximation of Eq. (8), as seen in Figs. 2 and 3. Hence, all the numerical values presented in this paper will correspond to $n_{\max }=64$. The Legendre polynomial as a function of $\cos \Theta$ is transformed to the frame of reference drawn in Fig. 1 using the addition theorem (e.g., Eq. (12.201) of Arfken, 1970)

$P_{n}(\cos \Theta)=P_{n}\left(\cos \theta_{*}\right) P_{n}(\cos \theta)+2 \sum_{m=1}^{n} \frac{(n-m) !}{(n+m) !} P_{n}^{m}\left(\cos \theta_{*}\right) P_{n}^{m}(\cos \theta) \cos (m \phi)$,

where $\theta_{*}$ is the angle between the direction of the central star from the center of the spinning body and the spin axis $\tilde{z}$. Thus, we get

$$
\Gamma(\cos \Theta)=\sum_{n=0}^{n_{\max }} \Gamma_{n 0} P_{n}(\cos \theta)+\Re\left[\sum_{n=1}^{n_{\max }} \sum_{m=1}^{n} \Gamma_{n m} P_{n}^{m}(\cos \theta) \exp (i m \phi)\right],
$$

where

$$
\Gamma_{n 0}=\Gamma_{n} P_{n}\left(\cos \theta_{*}\right),
$$

and

$$
\Gamma_{n m}=2 \Gamma_{n} \frac{(n-m) !}{(n+m) !} P_{n}^{m}\left(\cos \theta_{*}\right) .
$$

Next, we expand the second term of the RHS of Eq. $(23), \Lambda^{(j-1)}(\theta, \phi)$, using spherical harmonics:

$$
\Lambda^{(j)}(\theta, \phi)=\sum_{n=0}^{n_{\max }} \Lambda_{n 0}^{(j)} P_{n}(\cos \theta)+\Re\left[\sum_{n=1}^{n_{\max }} \sum_{m=1}^{n} \Lambda_{n m}^{(j)} P_{n}^{m}(\cos \theta) \exp (i m \phi)\right]
$$

where $\Lambda_{n 0}^{(j)}$ is a real number and $\Lambda_{n m}^{(j)}$ is a complex number for $m \geq 1$. These coefficients are obtained by using the orthogonal relations of the associated Legendre function (see Eqs. (8.14.11) and (8.14.13) of Abramowitz and Stegun (1964)):

$$
\Lambda_{n 0}^{(j)}=\frac{2 n+1}{4 \pi} \int_{-1}^{1} d \cos \theta P_{n}(\cos \theta) \int_{-\pi}^{\pi} d \phi \Lambda^{(j)}(\theta, \phi),
$$


and

$$
\Lambda_{n m}^{(j)}=\frac{2 n+1}{2 \pi} \frac{(n-m) !}{(n+m) !} \int_{-1}^{1} d \cos \theta P_{n}^{m}(\cos \theta) \int_{-\pi}^{\pi} d \phi \Lambda^{(j)}(\theta, \phi) \exp (-i m \phi) .
$$

Note that the denominator of the first term on the RHS of Eq. (34) is $2 \pi$ and not $4 \pi$, because we only use the terms with $m \geq 1$. Further, note that superscript $j$ is used in Eqs. (32) - (34) to represent general relations, but $j$ should be replaced with $j-1$ when Eqs. (33) and (34) are used in Eq. (23). We perform these integrals numerically by the method of quadrature by parts.

Thus, using spherical harmonics, we can expand all the three terms on the RHS of Eq. (23). By comparing terms with $m=0$ and $m \neq 0$, we get

$$
\delta C_{n}^{(j)}=f_{\text {relax }} \frac{\Gamma_{n 0}-\Lambda_{n 0}^{(j-1)}-q_{g l o b a l} n C_{n}^{(j-1)}}{1+q_{\text {global }} n},
$$

and

$$
\delta \mathcal{E}_{n m}^{(j)}=f_{\text {relax }} \frac{\Gamma_{n m}-\Lambda_{n m}^{(j-1)}-q_{\text {global }} \mathcal{E}_{n m}^{(j-1)} \hat{j}_{n, m}^{\prime}\left(\beta_{m}\right)}{\hat{j}_{n, m}\left(\beta_{m}\right)+q_{\text {global }} \hat{j}_{n, m}^{\prime}\left(\beta_{m}\right)},
$$

where $C_{n}^{(j-1)}=\sum_{\ell=1}^{j-1} \delta C_{n}^{(\ell)}$ and $\mathcal{E}_{n m}^{(j-1)}=\sum_{\ell=1}^{j-1} \delta \mathcal{E}_{n m}^{(\ell)}$. The factor $f_{\text {relax }}$ should be equal to unity in order to satisfy Eq. (23). If $\delta T^{(j)}$ which satisfy Eq. (23) is on the order of $\varepsilon^{j}$ with $\varepsilon<1$, the summation $\sum_{0}^{\infty} \delta T^{(j)}$ would converges. In practice, we use $f_{\text {relax }}=1$ at first to calculate the summation; if the summation converges, the converged value is considered the nonlinear solution which we wish to obtain (i.e., we empirically know that $\varepsilon<1$ ). However, if the summation does not converge, and just oscillate around a value (see Fig. 17 ), we empirically know that $\varepsilon \approx 1$. In this case, the values of $\delta C_{n}^{(j)}$ and $\delta \mathcal{E}_{n m}^{(j)}$ given by Eqs. (35) and (36), respectively, with $f_{\text {relax }}=1$ are too large 
for reducing the error of the nonlinear boundary condition given by Eq. (7); then we use a value of $f_{\text {relax }}$ smaller than unity in order for $\varepsilon$ to be smaller than unity. We could always find an appropriate value of $f_{\text {relax }}$ empirically to attenuate oscillations; we have confirmed that the converged solution satisfies the nonlinear boundary condition within a sufficiently small error. In principle, we can obtain a solution that satisfies the nonlinear boundary condition with an arbitrary accuracy by using a large value of $n_{\max }$, using a large number of meshes for performing numerical integration of Eqs. (33) and (34), and repeatedly using the abovementioned recurrence formulas as many times as needed.

\section{Results and Discussion}

The dimensionless temperature distribution (i.e., the dimensional temperature distribution normalized by the average temperature) is determined on the basis of the following three dimensionless parameters: (1) $q_{\text {global }}$, which indicates a characteristic value of the global conductive flux relative to the stellar heating rate, (2) $\omega$, the dimensionless angular velocity, which shows the angle of spin during the thermometric diffusion time $\tilde{R}^{2} / \tilde{\kappa}$, and (3) $\theta_{*}$, the angle between the direction of the central star and the spin axis of a body.

Although, these three parameters are sufficient for mathematically determining a solution, the thermal parameter, defined as $q_{\text {thermal }} \equiv\left(\tilde{k}_{T} \tilde{T}_{a v} / \tilde{l}_{\text {thermal }}\right) /\left(\alpha \tilde{F}_{*}\right)$, is often used to characterize the solution; this parameter indicates the ratio of the thermal energy stored in the surface region whose depth is equal to the penetration depth $\tilde{l}_{\text {thermal }}\left(\equiv(\tilde{\kappa} / \tilde{\omega})^{1 / 2}\right)$ of the thermal wave and the sur- 
face energy input (or output) by the stellar flux (or radiative loss) during a spin period (Farinella et al., 1998; Spencer et al., 1989; Vokrouhlický, 1998). Note that the thermal parameter defined in our study is $2^{-1 / 2}$ times that of Vokrouhlický (1998), because we use the global average temperature $\tilde{T}_{a v}$ instead of the temperature of the point where the stellar direction is perpendicular to the surface of the body, $\tilde{T}_{*} \equiv\left(\alpha \tilde{F}_{*} / \epsilon \tilde{\sigma}\right)^{1 / 4}$, in the definition of the thermal parameter. Since $\omega=\left(q_{\text {thermal }} / q_{\text {global }}\right)^{2}$, the dimensionless angular velocity $\omega$ can be determined if $q_{\text {thermal }}$ and $q_{\text {global }}$ are known; therefore, $q_{\text {thermal }}, q_{\text {global }}$, and $\theta_{*}$ are used as a parameter set for characterizing a solution. Note that $\omega^{-1 / 2}=q_{\text {global }} / q_{\text {thermal }}=\tilde{l}_{\text {thermal }} / \tilde{R}$ gives a measure of the importance of $3 \mathrm{D}$ heat conduction relative to $1 \mathrm{D}$ heat conduction.

\section{Figures $4-6$}

Figures $4-6$ show the longitudinal distributions of the temperature at two colatitudes $\theta=60^{\circ}$ and $120^{\circ}$ for $(\mathrm{A}) q_{\text {global }}=0.1$ and $q_{\text {thermal }}=0.1$, (B) $q_{\text {global }}=0.1$ and $q_{\text {thermal }}=1$, and $(\mathrm{C}) q_{\text {global }}=0.1$ and $q_{\text {thermal }}=10$, with $\theta_{*}=60^{\circ}$. The solid lines in Fig. 4 show the nonlinear solution obtained by the iterative method for $j=32$ and $f_{\text {relax }}=0.5$, and the ones in Figs. 5 and 6 show the values for $j=16$ and $f_{\text {relax }}=1.0$; the dashed lines show the linear solution (i.e., $j=1$ and $f_{\text {relax }}=1.0$ ). In general, the temperature according to a nonlinear solution is lower than the temperature according to a linear solution at a same position. This is because the dimensionless form of the cooling rate $T^{4} / 4=(1+\delta T)^{4} / 4$ used to determine the nonlinear solution (see Eq. (7)) is larger than $(1 / 4)+\delta T$ used to determine the linear solution.

Figures $7-9$ 
Figures $7-9$ show the maximum and minimum temperatures at each colatitude for parameter sets $(\mathrm{A})-(\mathrm{C})$ in Figs. $4-6$, respectively. The difference between the maximum and minimum temperatures is large and $T_{\min }^{(j)} \lesssim 0.6$ over a wide range of $\theta$ for $(\mathrm{A})$, as seen in Fig. 7 . On the other hand, the difference between the maximum and minimum temperatures is small and $T^{(j)} \sim 0.6$ only in a region experiencing the polar night (i.e., $\left.\theta \geq \theta_{*}+90^{\circ}=150^{\circ}\right)$ for $(\mathrm{C})$ as seen in Fig. 9.

Figures $10-12$

Figures 10 - 12 show the longitude of the maximum- and minimumtemperature points at each colatitude for parameter sets $(A)-(C)$ in Figs. $4-6$, respectively. The longitudes of the maximum-temperature points are nearly equal to 0 (i.e., at noon) for (A), as seen in Fig. 10; the angular velocity is very low, so the thermal energy given by the stellar irradiation is re-emitted before the body spins substantially. On the other hand, the longitude of the maximum-temperature point is in the afternoon for $(\mathrm{B})$ and $(\mathrm{C})$, as seen in Figs. 11 and 12, respectively, because of moderate to high values of angular velocities. The longitude of the minimum temperature point is not shown in Fig. 10 because it is difficult to determine that point due to flat temperature distributions where it is night (see Fig. 4). The longitude of the minimumtemperature point for (B) and (C) in Figs. 11 and 12, respectively, are nearly equal to the longitude of sunup, because radiative cooling continues until the sunup. However, for $\theta<90^{\circ}-\theta_{*}=30^{\circ}$, i.e., the region experiencing the midnight sun, the longitude of the minimum-temperature point is about $180^{\circ}$ from the longitude of the maximum-temperature point. The longitudes of the maximum and minimum temperature points at each colatitude according to 
both the linear and nonlinear solutions are almost identical. The temperature distribution for $\theta>90^{\circ}+\theta_{*}=150^{\circ}$ (i.e., the region experiencing the polar night) is very flat and we do not plot the longitudes of the maximum- and minimum-temperature points in this region.

\section{Table 1}

We calculated the temperature distribution for the parameter sets $\left(q_{\text {global }}, q_{\text {global }}, \theta_{*}\right)$ listed in Table 1. The maximum and minimum temperatures on a sphere determined by performing the $j$-th iteration and denoted as $T_{\max }^{(j)}$ and $T_{\min }^{(j)}$, respectively, are listed in Table 1 . The maximum and minimum temperatures according to the linear solution, denoted as $T_{\max }^{(1)}$ and $T_{\min }^{(1)}$, respectively, determined by using $j=1$ and $f_{\text {relax }}=1$ are also listed for comparison. When $q_{\text {global }} \ll 1$, the values of the maximum and minimum temperatures differ significantly from unity; on the other hand, when $q_{\text {global }} \gg 1$, the values of the maximum and minimum temperatures are nearly equal to unity.

\section{Table 2}

The colatitude and longitude of the maximum-temperature point, denoted as $\theta_{\max }^{(j)}$ and $\phi_{\max }^{(j)}$, respectively, are shown in Table 2 . It is difficult to determine the position at which the temperature is $T_{\text {min }}^{(j)}$ because the temperature distribution is very flat at $T \approx T_{\text {min }}^{(j)}$. The colatitude and longitude of the maximum-temperature point in the case of the linear solution $\left(\theta_{\max }^{(1)}, \phi_{\max }^{(1)}\right)$ are also shown in Table 2. The coordinates of the maximum-temperature points are almost the same in the case of the iterative and linear solutions. When $q_{\text {thermal }} \ll 1$, the maximum-temperature point $\left(\theta_{\max }, \phi_{\max }\right)$ is near 
the point $\left(\theta_{*}, 0\right)$ where the stellar direction is perpendicular to the surface of the body. On the other hand, when $q_{\text {thermal }} \gg 1$, the maximum-temperature point is away from $\left(\theta_{*}, 0\right)$.

In order to understand the obtained results using dimensional variables, we introduce the critical radius $\tilde{R}_{c}$ at which $q_{g l o b a l}=1$,

$$
\tilde{R}_{c}=\frac{\tilde{k}_{T} \tilde{T}_{a v}}{\alpha \tilde{F}_{*}}=0.20\left(\frac{\tilde{a}}{1 \mathrm{AU}}\right)^{3 / 2}\left(\frac{\tilde{L}_{*}}{\tilde{L}_{\odot}}\right)^{-3 / 4} \alpha^{-3 / 4} \epsilon^{-1 / 4}\left(\frac{\tilde{k}_{T}}{1 \mathrm{~W} \mathrm{~m}^{-1} \mathrm{~K}^{-1}}\right)[\mathrm{m}],
$$

and the critical spin period $\tilde{P}_{c}$ at which $q_{\text {thermal }}=1$,

$$
\begin{aligned}
\tilde{P}_{c}= & \frac{2 \pi \tilde{T}_{a v}^{2} \tilde{k}_{T}^{2}}{\alpha^{2} \tilde{F}_{*}^{2} \tilde{\kappa}} \\
= & 2.6 \times 10^{5}\left(\frac{\tilde{a}}{1 \mathrm{AU}}\right)^{3}\left(\frac{\tilde{L}_{*}}{\tilde{L}_{\odot}}\right)^{-3 / 2} \alpha^{-3 / 2} \epsilon^{-1 / 2} \\
& \left(\frac{\tilde{\kappa}}{1 \times 10^{-6} \mathrm{~m}^{2} \mathrm{~s}^{-1}}\right)^{-1}\left(\frac{\tilde{k}_{T}}{1 \mathrm{~W} \mathrm{~m}^{-1} \mathrm{~K}^{-1}}\right)^{2}[\mathrm{~s}],
\end{aligned}
$$

where the material constants $\tilde{\kappa}$ and $\tilde{k}_{T}$ are normalized by the typical values of ordinary chondrites (Opeil et al., 2010; Yomogida and Matsui, 1983). By using these values, the dimensionless parameters are expressed as

$$
q_{g l o b a l}=\tilde{R}_{c} / \tilde{R}
$$

and

$$
q_{\text {thermal }}=\left(\tilde{P}_{c} / \tilde{P}\right)^{1 / 2}
$$

If $q_{\text {global }} \gg 1$, i.e., $\tilde{R} \ll \tilde{R}_{c}$, the thermal conduction is so effective that the temperature is nearly constant throughout the sphere (see Table 1). On the other hand, if $q_{\text {global }} \ll 1$, i.e., $\tilde{R} \gg \tilde{R}_{c}$, the maximum and minimum 
temperatures differ considerably from the average temperature (see Table 1). If $q_{\text {thermal }} \gg 1$, i.e., $\tilde{P} \ll \tilde{P}_{c}$, the temperature is averaged over the longitude $\phi$ to be nearly axisymmetric with respect to the spin axis $z$, because of the fast spin, even though the dependence of temperature on colatitudes remains if $q_{\text {global }} \lesssim 1$ (see Figs. 6 and 9 ). On the other hand, if $q_{\text {thermal }} \ll 1$ and $q_{\text {global }} \lesssim 1$, i.e., $\tilde{P} \gg \tilde{P}_{c}$ and $\tilde{R} \gtrsim \tilde{R}_{c}$, the temperature would be high in the region where it is day and low in the region where it is night (see Fig. 4). In the studies of the diurnal Yarkovsky effect, the most interesting case is when $q_{\text {thermal }} \gtrsim 1$ and $q_{\text {global }} \lesssim 1$. Then, the delays in temperature variation occurs because of the thermal inertia, and as a result, the maximum temperature is reached in the afternoon (see Table 2) (Farinella et al., 1998). This delay causes the diurnal Yarkovsky effect.

We have used some values of $q_{\text {global }}$ and $q_{\text {thermal }}$ for reference (see Tables 1 - 2). The values $q_{\text {global }}=0.1,1$, and 10 correspond to $\tilde{R}=2 \mathrm{~m}, 20 \mathrm{~cm}$ and $2 \mathrm{~cm}$, respectively, and $q_{\text {thermal }}=0.1,1$ and 10 correspond to $\tilde{P}=3 \times 10^{7} \mathrm{~s}, 3 \times 10^{5} \mathrm{~s}$ and $3 \times 10^{3}$ s, respectively, at $1 \mathrm{AU}$ for standard values of parameters: $\tilde{L}_{*}=1$ $\tilde{L}_{\odot}, \alpha=1, \epsilon=1, \tilde{\kappa}=1 \times 10^{-6} \mathrm{~m}^{2} \mathrm{~s}^{-1}$ and $\tilde{k}_{T}=1 \mathrm{~W} \mathrm{~m}^{-1} \mathrm{~K}^{-1}$. Kadono et al. (2009) determined the spin periods of asteroid fragments as $\tilde{P}[\mathrm{~s}] \sim$ $20 \tilde{R}[\mathrm{~m}]$ from impact-disruption experiments of thin glass plates. By using this relation with Eqs. (39) and (40), and the standard values of parameters, we have $q_{\text {thermal }} \sim 3 \times 10^{2}(\tilde{a} / 1 \mathrm{AU})^{3 / 4} q_{\text {global }}^{1 / 2}$. Hence, we get $q_{\text {thermal }} \gg 1$ for meter- to centimeter-sized meteoroids with $q_{g l o b a l} \sim 1$, although this result is not conclusive because it is uncertain whether meteoroids rotates with the relation determined by Kadono et al. (2009) and also because meteoroids with large values of the porosity may have values of the thermal conductivity 
and the thermometric conductivity very different from the standard values.

\section{Table 3}

The force due to the back reaction of the radiation from a unit surface of a body is given by the following equation:

$$
\tilde{\boldsymbol{P}}_{\text {rad }}=-\frac{2}{3} \epsilon \frac{\tilde{\sigma}[\tilde{T}(\theta, \phi)]^{4}}{\tilde{c}} \boldsymbol{n}=-\frac{2 \alpha \tilde{F}_{*}}{3 \tilde{c}} \Lambda(\theta, \phi) \boldsymbol{n},
$$

where $\tilde{c}$ is the speed of light and $\boldsymbol{n}$ is the unit vector normal to the surface of the body. By substituting Eq. (32) into this equation and integrating over the surface of the spherical body, we estimate the force acting on the body for the temperature distribution determined by the $j$-th iteration:

$$
\tilde{\boldsymbol{f}}^{(j)}=\frac{8 \pi \tilde{R}^{2} \alpha \tilde{F}_{*}}{9 \tilde{c}}\left\{-\Re\left[\Lambda_{1,1}^{(j)}\right] \boldsymbol{i}+\Im\left[\Lambda_{1,1}^{(j)}\right] \boldsymbol{j}-\Lambda_{1,0}^{(j)} \boldsymbol{k}\right\}
$$

where $\Re$ and $\Im$ denote the real and imaginary parts, respectively, and $\boldsymbol{i}, \boldsymbol{j}$, and $\boldsymbol{k}$ denote unit vectors in the directions of $x, y$, and $z$ axes respectively. The rate of change in the semimajor axis is proportional to $f_{y}$ (Vokrouhlický, 1998). In Table $3, f_{y}^{(j)}$, the $y$-component of the force determined by the $j$-th iteration normalized by $4 \pi \tilde{R}^{2} \alpha \tilde{F}_{*} /(9 \tilde{c})$, for the parameter sets listed in Tables 1 and 2 is listed. The force determined by the linear approximation, $f_{y, \text { linear }}$, is also listed in Table 3 for comparison; we calculated $f_{y, \text { linear }}$ by using an approximation of the dimensionless radiative cooling rate: $\Lambda^{(1)}=\left[T^{(1)}\right]^{4} / 4 \approx(1 / 4)+\delta T^{(1)}$, where $\delta T^{(1)}$ is determined by using $f_{\text {relax }}=1$. We have confirmed that $f_{y, \text { linear }}$ is identical to the force determined by Vokrouhlický (1998).

It is seen that the maximum difference between the results of the iterative method and those of linear approximation is $13 \%$ (this percentage is 
for $q_{\text {global }}=0.1$ and $q_{\text {thermal }}=10$ ) and that the nonlinear effect does not significantly affect the diurnal Yarkovsky effect. However, precise temperature distributions are useful for observational astronomers. Moreover, our results would be useful for checking the accuracy of complicated computer codes that calculate the surface temperature of a spinning body irradiated by a central star.

In order to examine the convergence of the iterative method, we calculated the RMS error of the boundary condition (see Eq. (7)); the RMS error is defined as

$$
\Delta^{(j)} \equiv\left\{\frac{1}{4 \pi} \int_{-1}^{1} d \cos \theta \int_{-\pi}^{\pi} d \phi\left[\Gamma(\cos \Theta)-\Lambda^{(j)}-\left.q_{g l o b a l} \frac{\partial T^{(j)}}{\partial r}\right|_{r=1}\right]^{2}\right\}^{1 / 2}
$$

Figures $13-15$

Figures 13 - 15 respectively show the RMS error $\Delta$ as a function of the iteration frequency $j$ for the following three cases: (a) $\theta_{*}=60^{\circ}, q_{\text {global }}=0.1$, $q_{\text {thermal }}=1.0$, and $f_{\text {relax }}=1.0$, (b) $\theta_{*}=60^{\circ}, q_{\text {global }}=0.1, q_{\text {thermal }}=0.1$, and $f_{\text {relax }}=1.0$, and $(\mathrm{c}) \theta_{*}=60^{\circ}, q_{\text {global }}=0.1, q_{\text {thermal }}=0.1$, and $f_{\text {relax }}=0.5$. In these calculations, we set $n_{\max }=64$, and the numerical integration by $\theta$ and $\phi$ in Eqs. (33) and (34) are performed using 1440 and 2880 meshes, respectively. Figure 13 shows that the RMS error becomes less than $10^{-3}$ after 14 iterations in case (a). On the other hand, Fig. 14 shows that the RMS error does not converge in case (b) in which $f_{\text {relax }}=1.0$. By using same values of $q_{\text {global }}$ and $q_{\text {thermal }}$ as Fig. 14 , but by changing the value of $f_{\text {relax }}$ to 0.5 , the RMS error becomes less than $10^{-3}$ after 30 iterations, as seen in Fig. 15 . 
Figures $16-18$

In order to understand why the RMS error does not converge in case (b), we plot the global maximum temperature $T_{\max }^{(j)}$ as a function of $j$ for cases (a) - (c) in Figs. $16-18$, respectively. As seen in Fig. 16, $T_{\max }^{(j)}$ converges to a limiting value in case (a). On the other hand, Fig. 17 shows that $T_{\max }^{(j)}$ oscillates around a limiting value and does not converge in case (b). The spin is so slow in case (b) that the maximum temperature around the point where the stellar direction is perpendicular to the surface of the body differs considerably from unity and the correction of each iteration step becomes also too large to converge. This problem has been overcome by using $f_{\text {relax }}=0.5$, as seen in Fig. 18.

If we perform a sufficient number of iterations and use an appropriate value of $f_{\text {relax }}$, a sufficient number of meshes for the integration in Eqs. (33) and (34), and a sufficient number of $n_{\max }$ for the spherical harmonics expansion, we can, in principle, obtain a solution within any desired value of the RMS error.

\section{Conclusions}

In this work, we have developed an iterative method for determining the temperature distribution in a spherical body that is irradiated by a central star. We have taken into account the spin only and have neglected the seasonal revolution. It is assumed that material parameters such as the thermal conductivity and the thermometric conductivity remain constant throughout a spherical body. 
A general solution for the temperature distribution inside a body had been obtained using spherical harmonics and spherical Bessel functions by Vokrouhlický (1998). However, the surface boundary condition contains a term obtained using the Stefan-Boltzmann law and is nonlinear with respect to the temperature because it is dependent on the fourth power of temperature. An unresolved problem was to fit the solution to the nonlinear boundary condition at the surface. An iterative method is used to calculate the values of the constant coefficients of the general solution.

A temperature distribution is characterized by the following three dimensionless parameters: (1) $q_{\text {global }}$, which indicates the ratio of the conductive flux for the temperature gradient given by the average temperature divided by the radius of the body and the stellar heating rate (or the radiative cooling rate), (2) $q_{\text {thermal }}$, which indicates the ratio of the thermal energy stored in the surface region whose depth is equal to the penetration depth of the thermal wave and the surface energy input (or output) by the absorbed stellar flux (or radiative loss) during a spin period, and (3) $\theta_{*}$, the angle between the direction of the central star from the center of the spinning body and the spin axis of a body.

The value of $q_{g l o b a l}$ is inversely proportional to the radius of the body. If the radius of the body is large such that $q_{\text {global }} \ll 1$, thermal conduction is not globally effective. In this case, the temperature distribution obtained by the iterative method is very different from that obtained by the linear approximation of radiative cooling.

The value of $q_{\text {thermal }}$ is inversely proportional to the square root of the spin period. If the spin period is short such that $q_{\text {thermal }} \gg 1$, the temperature 
becomes almost constant and independent of the longitude for a given value of the colatitude as a result of the fast spin.

The force that changes the semimajor axis due to the diurnal Yarkovsky effect is determined. The maximum difference between the force according to the iterative solution and that according to the linear solution is about 10\%; the nonlinear effect does not significantly affect the diurnal Yarkovsky effect.

However, the surface temperature for a large body with $q_{\text {global }} \ll 1$, which would be important for the observational astronomers, is very different from the surface temperature according to the linear solution. This method would be a base of three dimensional calculations of thermal histories of solar system bodies as well; any numerical codes that calculate three-dimensional temperature evolutions in spherical bodies can be checked by using the nonlinear solution obtained in this study. 


\section{Figure Captions}

Fig. 1. The spherical coordinates. The spin axis is $\tilde{z}$, and the direction of the central star is in the $\tilde{x}-\tilde{z}$ plane and it is at an angle $\theta_{*}$ from the $\tilde{z}$ axis. The radius of the body is $\tilde{R}$. The angle between the stellar direction and a point $\mathrm{P}$ with coordinates $(\tilde{R}, \theta, \phi)$ when viewed from the center of the body is $\Theta$.

Fig. 2. The dimensionless stellar heating rate $\Gamma(\cos \Theta)$ expressed by the Legendre expansions with $0 \leq n \leq 16$ (dotted line) and $0 \leq n \leq 64$ (solid line).

Fig. 3. The error of $\Gamma(\cos \Theta)$ for $0 \leq n \leq 16$ (dotted line) and $0 \leq n \leq 64$ (solid line).

Fig. 4. The longitudinal distributions of the temperature at two colatitudes $\theta=60^{\circ}$ and $120^{\circ}$ for $(\mathrm{A}) q_{\text {global }}=0.1, q_{\text {thermal }}=0.1$, and $\theta_{*}=60^{\circ}$. The solid lines show the values obtained by the iterative method with $j=32$ and $f_{\text {relax }}=0.5$; the dashed lines show the values obtained by the linear method (i.e., $j=1$ and $f_{\text {relax }}=1.0$ ). The longitudes of sunup and sundown, respectively, are $-109.5^{\circ}$ and $109.5^{\circ}$ for $\theta=60^{\circ}$, and $-70.5^{\circ}$ and $70.5^{\circ}$ for $\theta=120^{\circ}$.

Fig. 5. The longitudinal distribution of the temperature at two colatitudes $\theta=60^{\circ}$ and $120^{\circ}$ for $(\mathrm{B}) q_{\text {global }}=0.1, q_{\text {thermal }}=1$, and $\theta_{*}=60^{\circ}$. The solid lines show the values obtained by the iterative method with $j=16$ and $f_{\text {relax }}=1.0$; the dashed lines show the values obtained by the linear method (i.e., $j=1$ and $f_{\text {relax }}=1.0$ ). The longitudes of sunup and sundown are same as those in Fig. 4.

Fig. 6. The longitudinal distribution of the temperature at two colatitudes 
$\theta=60^{\circ}$ and $120^{\circ}$ for $(\mathrm{C}) q_{\text {global }}=0.1, q_{\text {thermal }}=10$, and $\theta_{*}=60^{\circ}$. The solid lines show the values obtained by the iterative method with $j=16$ and $f_{\text {relax }}=1.0$; the dashed lines show the values obtained by the linear method (i.e., $j=1$ and $f_{\text {relax }}=1.0$ ). The longitudes of sunup and sundown are same as those in Fig. 4.

Fig. 7. The maximum and minimum temperatures at each colatitude for (A) $q_{\text {global }}=0.1, q_{\text {thermal }}=0.1$, and $\theta_{*}=60^{\circ}$. The solid lines show the values obtained by the iterative method with $j=32$ and $f_{\text {relax }}=0.5$; the dashed lines show the values obtained by the linear method (i.e., $j=1$ and $\left.f_{\text {relax }}=1.0\right)$.

Fig. 8. The maximum and minimum temperatures at each colatitude for (B) $q_{\text {global }}=0.1, q_{\text {thermal }}=1$, and $\theta_{*}=60^{\circ}$. The solid lines show the values obtained by the iterative method with $j=16$ and $f_{\text {relax }}=1.0$; the dashed lines show the values obtained by the linear method (i.e., $j=1$ and $\left.f_{\text {relax }}=1.0\right)$.

Fig. 9. The maximum and minimum temperatures at each colatitude for (C) $q_{\text {global }}=0.1, q_{\text {thermal }}=10$, and $\theta_{*}=60^{\circ}$. The solid lines show the values obtained by the iterative method with $j=16$ and $f_{\text {relax }}=1.0$; the dashed lines show the values obtained by the linear method (i.e., $j=1$ and $\left.f_{\text {relax }}=1.0\right)$.

Fig. 10. The longitude of the maximum-temperature point at each colatitude for $(\mathrm{A}) q_{\text {global }}=0.1, q_{\text {thermal }}=0.1$, and $\theta_{*}=60^{\circ}$. The solid line shows the values obtained by the iterative method with $j=32$ and $f_{\text {relax }}=0.5$; the dashed line shows the values obtained by the linear method (i.e., $j=1$ and $\left.f_{\text {relax }}=1.0\right)$. 
Fig. 11. The longitudes of the maximum- and minimum-temperature points at each colatitude for $(\mathrm{B}) q_{\text {global }}=0.1, q_{\text {thermal }}=1$, and $\theta_{*}=60^{\circ}$. The solid lines show the values obtained by the iterative method with $j=16$ and $f_{\text {relax }}=1.0$; the dashed lines show the values obtained by the linear method (i.e., $j=1$ and $f_{\text {relax }}=1.0$ ). The dashed-dotted line shows the longitude of sunup.

Fig. 12. The longitudes of the maximum- and minimum-temperature points at each colatitude for $(\mathrm{C}) q_{\text {global }}=0.1, q_{\text {thermal }}=10$, and $\theta_{*}=60^{\circ}$. The solid lines show the values obtained by the iterative method with $j=16$ and $f_{\text {relax }}=1.0$; the dashed lines show the values obtained by the linear method (i.e., $j=1$ and $f_{\text {relax }}=1.0$ ). The dashed-dotted line shows the longitude of sunup.

Fig. 13. RMS error $\Delta$ as a function of the iteration frequency $j$ when (a) $\theta_{*}=60^{\circ}, q_{\text {global }}=0.1, q_{\text {thermal }}=1.0$, and $f_{\text {relax }}=1.0$.

Fig. 14. RMS error $\Delta$ as a function of the iteration frequency $j$ when (b) $\theta_{*}=60^{\circ}, q_{\text {global }}=0.1, q_{\text {thermal }}=0.1$, and $f_{\text {relax }}=1.0$.

Fig. 15. RMS error $\Delta$ as a function of the iteration frequency $j$ when (c) $\theta_{*}=60^{\circ}, q_{\text {global }}=0.1, q_{\text {thermal }}=0.1$, and $f_{\text {relax }}=0.5$.

Fig. 16. The global maximum temperature $T_{m a x}^{(j)}$ as a function of the iteration frequency $j$ when (a) $\theta_{*}=60^{\circ}, q_{\text {global }}=0.1, q_{\text {thermal }}=1.0$, and $f_{\text {relax }}=1.0$.

Fig. 17. The global maximum temperature $T_{\max }^{(j)}$ as a function of the iteration frequency $j$ when (b) $\theta_{*}=60^{\circ}, q_{\text {global }}=0.1, q_{\text {thermal }}=0.1$, and $f_{\text {relax }}=1.0$.

Fig. 18. The global maximum temperature $T_{\max }^{(j)}$ as a function of the 
iteration frequency $j$ when (c) $\theta_{*}=60^{\circ}, q_{\text {global }}=0.1, q_{\text {thermal }}=0.1$, and $f_{\text {relax }}=0.5$. 
Table 1: The maximum and minimum temperatures according to iterative solutions and linear solutions. A solution is characterized by three dimensionless parameters: $q_{g l o b a l}$, $q_{\text {thermal }}$, and $\theta_{*}$. The maximum and minimum temperatures according to a solution determined by performing the $j$-th iteration are denoted as $T_{m a x}^{(j)}$ and $T_{\min }^{(j)}$, respectively, and $f_{\text {relax }}$ is relaxation parameter used in Eqs. (35) and (36). The maximum and minimum temperatures according to a linear solution are denoted as $T_{\max }^{(1)}$ and $T_{\min }^{(1)}$, respectively.

\begin{tabular}{rrrrrrrrc}
\hline$q_{\text {global }}$ & $q_{\text {thermal }}$ & $\theta_{*}$ & $j$ & $f_{\text {relax }}$ & $T_{\max }^{(j)}$ & $T_{\max }^{(1)}$ & $T_{\min }^{(j)}$ & $T_{\min }^{(1)}$ \\
\hline 0.1 & 0.1 & $30^{\circ}$ & 32 & 0.5 & 1.397 & 1.668 & 0.527 & 0.757 \\
0.1 & 1.0 & $30^{\circ}$ & 32 & 0.5 & 1.380 & 1.599 & 0.548 & 0.760 \\
0.1 & 10 & $30^{\circ}$ & 32 & 0.5 & 1.350 & 1.549 & 0.552 & 0.761 \\
1.0 & 0.1 & $30^{\circ}$ & 16 & 1.0 & 1.274 & 1.340 & 0.800 & 0.840 \\
1.0 & 1.0 & $30^{\circ}$ & 16 & 1.0 & 1.274 & 1.340 & 0.800 & 0.840 \\
1.0 & 10 & $30^{\circ}$ & 16 & 1.0 & 1.236 & 1.283 & 0.814 & 0.846 \\
10 & 0.1 & $30^{\circ}$ & 16 & 1.0 & 1.057 & 1.059 & 0.966 & 0.968 \\
10 & 1.0 & $30^{\circ}$ & 16 & 1.0 & 1.057 & 1.059 & 0.966 & 0.968 \\
10 & 10 & $30^{\circ}$ & 16 & 1.0 & 1.057 & 1.057 & 0.967 & 0.968 \\
0.1 & 0.1 & $60^{\circ}$ & 32 & 0.5 & 1.397 & 1.668 & 0.528 & 0.756 \\
0.1 & 1.0 & $60^{\circ}$ & 16 & 1.0 & 1.333 & 1.466 & 0.604 & 0.768 \\
0.1 & 10 & $60^{\circ}$ & 16 & 1.0 & 1.186 & 1.230 & 0.614 & 0.767 \\
1.0 & 0.1 & $60^{\circ}$ & 16 & 1.0 & 1.274 & 1.340 & 0.799 & 0.840 \\
1.0 & 1.0 & $60^{\circ}$ & 16 & 1.0 & 1.273 & 1.338 & 0.800 & 0.840 \\
1.0 & 10 & $60^{\circ}$ & 16 & 1.0 & 1.131 & 1.142 & 0.853 & 0.855 \\
10 & 0.1 & $60^{\circ}$ & 16 & 1.0 & 1.057 & 1.059 & 0.966 & 0.968 \\
10 & 1.0 & $60^{\circ}$ & 16 & 1.0 & 1.057 & 1.059 & 0.966 & 0.968 \\
10 & 10 & $60^{\circ}$ & 16 & 1.0 & 1.056 & 1.058 & 0.967 & 0.968 \\
0.1 & 0.1 & $90^{\circ}$ & 32 & 0.5 & 1.397 & 1.668 & 0.528 & 0.756 \\
0.1 & 1.0 & $90^{\circ}$ & 16 & 1.0 & 1.315 & 1.429 & 0.731 & 0.803 \\
0.1 & 10 & $90^{\circ}$ & 16 & 1.0 & 1.110 & 1.117 & 0.772 & 0.819 \\
1.0 & 0.1 & $90^{\circ}$ & 16 & 1.0 & 1.274 & 1.340 & 0.799 & 0.840 \\
1.0 & 1.0 & $90^{\circ}$ & 16 & 1.0 & 1.273 & 1.338 & 0.801 & 0.841 \\
1.0 & 10 & $90^{\circ}$ & 16 & 1.0 & 1.084 & 1.086 & 0.930 & 0.933 \\
10 & 0.1 & $90^{\circ}$ & 16 & 1.0 & 1.057 & 1.059 & 0.966 & 0.968 \\
10 & 1.0 & $90^{\circ}$ & 16 & 1.0 & 1.057 & 1.059 & 0.966 & 0.968 \\
10 & 10 & $90^{\circ}$ & 16 & 1.0 & 1.056 & 1.057 & 0.967 & 0.968 \\
\hline & & & & & & & &
\end{tabular}


Table 2: Positions of the maximum-temperature points according to iterative solutions and linear solutions. Colatitude and longitude of the maximum-temperature point for a solution, which is determined by the $j$-th iteration are denoted as $\theta_{\max }^{(j)}$ and $\phi_{\max }^{(j)}$, respectively, while those for a linear solution are denoted as $\theta_{\max }^{(1)}$ and $\phi_{\max }^{(1)}$, respectively.

\begin{tabular}{rrrrrrrrr}
\hline$q_{\text {global }}$ & $q_{\text {thermal }}$ & $\theta_{*}$ & $j$ & $f_{\text {relax }}$ & $\theta_{\max }^{(j)}$ & $\theta_{\max }^{(1)}$ & $\phi_{\max }^{(j)}$ & $\phi_{\max }^{(1)}$ \\
\hline 0.1 & 0.1 & $30^{\circ}$ & 32 & 0.5 & 30.0 & 30.0 & 1.3 & 1.3 \\
0.1 & 1.0 & $30^{\circ}$ & 32 & 0.5 & 23.8 & 20.3 & 13.3 & 23.6 \\
0.1 & 10 & $30^{\circ}$ & 32 & 0.5 & 6.9 & 3.5 & 37.3 & 41.4 \\
1.0 & 0.1 & $30^{\circ}$ & 16 & 1.0 & 30.0 & 30.0 & 0.0 & 0.0 \\
1.0 & 1.0 & $30^{\circ}$ & 16 & 1.0 & 29.9 & 29.9 & 4.3 & 4.8 \\
1.0 & 10 & $30^{\circ}$ & 16 & 1.0 & 8.8 & 7.5 & 41.4 & 44.4 \\
10 & 0.1 & $30^{\circ}$ & 16 & 1.0 & 30.0 & 30.0 & 0.0 & 0.0 \\
10 & 1.0 & $30^{\circ}$ & 16 & 1.0 & 30.0 & 30.0 & 0.13 & 0.13 \\
10 & 10 & $30^{\circ}$ & 16 & 1.0 & 29.6 & 29.6 & 8.0 & 8.0 \\
0.1 & 0.1 & $60^{\circ}$ & 32 & 0.5 & 60.0 & 60.0 & 1.1 & 1.1 \\
0.1 & 1.0 & $60^{\circ}$ & 16 & 1.0 & 54.3 & 53.5 & 15.5 & 22.1 \\
0.1 & 10 & $60^{\circ}$ & 16 & 1.0 & 15.5 & 11.4 & 39.4 & 41.8 \\
1.0 & 0.1 & $60^{\circ}$ & 16 & 1.0 & 60.0 & 60.0 & 0.0 & 0.0 \\
1.0 & 1.0 & $60^{\circ}$ & 16 & 1.0 & 59.9 & 59.9 & 4.1 & 4.6 \\
1.0 & 10 & $60^{\circ}$ & 16 & 1.0 & 32.3 & 30.9 & 42.0 & 43.5 \\
10 & 0.1 & $60^{\circ}$ & 16 & 1.0 & 60.0 & 60.0 & 0.0 & 0.0 \\
10 & 1.0 & $60^{\circ}$ & 16 & 1.0 & 60.0 & 60.0 & 0.13 & 0.13 \\
10 & 10 & $60^{\circ}$ & 16 & 1.0 & 59.6 & 59.6 & 8.0 & 8.0 \\
0.1 & 0.1 & $90^{\circ}$ & 32 & 0.5 & 90.0 & 90.0 & 1.1 & 1.1 \\
0.1 & 1.0 & $90^{\circ}$ & 16 & 1.0 & 90.0 & 90.0 & 15.0 & 20.0 \\
0.1 & 10 & $90^{\circ}$ & 16 & 1.0 & 90.0 & 90.0 & 33.1 & 33.9 \\
1.0 & 0.1 & $90^{\circ}$ & 16 & 1.0 & 90.0 & 90.0 & 0.0 & 0.0 \\
1.0 & 1.0 & $90^{\circ}$ & 16 & 1.0 & 90.0 & 90.0 & 4.1 & 4.6 \\
1.0 & 10 & $90^{\circ}$ & 16 & 1.0 & 90.0 & 90.0 & 35.6 & 36.0 \\
10 & 0.1 & $90^{\circ}$ & 16 & 1.0 & 90.0 & 90.0 & 0.0 & 0.0 \\
10 & 1.0 & $90^{\circ}$ & 16 & 1.0 & 90.0 & 90.0 & 0.13 & 0.13 \\
10 & 10 & $90^{\circ}$ & 16 & 1.0 & 90.0 & 90.0 & 8.0 & 8.0 \\
\hline & & & & & & & &
\end{tabular}


Table 3: Forces due to the back reaction of radiation normalized by $4 \pi \tilde{R}^{2} \alpha \tilde{F}_{*} /(9 \tilde{c})$ for iterative solutions $f_{y}^{(j)}$ and linear solutions $f_{y \text {, linear }}$.

\begin{tabular}{rrrrrrr}
\hline$q_{\text {global }}$ & $q_{\text {thermal }}$ & $\theta_{*}$ & $j$ & $f_{\text {relax }}$ & $f_{y}^{(j)}$ & $f_{\text {y,linear }}$ \\
\hline 0.1 & 0.1 & $30^{\circ}$ & 32 & 0.5 & -0.0084 & -0.0082 \\
0.1 & 1.0 & $30^{\circ}$ & 32 & 0.5 & -0.1030 & -0.1130 \\
0.1 & 10 & $30^{\circ}$ & 32 & 0.5 & -0.0358 & -0.0311 \\
1.0 & 0.1 & $30^{\circ}$ & 16 & 1.0 & -0.0002 & -0.0003 \\
1.0 & 1.0 & $30^{\circ}$ & 16 & 1.0 & -0.0232 & -0.0246 \\
1.0 & 10 & $30^{\circ}$ & 16 & 1.0 & -0.0384 & -0.0350 \\
10 & 0.1 & $30^{\circ}$ & 16 & 1.0 & 0.0000 & 0.0000 \\
10 & 1.0 & $30^{\circ}$ & 16 & 1.0 & -0.0001 & -0.0001 \\
10 & 10 & $30^{\circ}$ & 16 & 1.0 & -0.0079 & -0.0079 \\
0.1 & 0.1 & $60^{\circ}$ & 32 & 0.5 & -0.0145 & -0.0142 \\
0.1 & 1.0 & $60^{\circ}$ & 16 & 1.0 & -0.1793 & -0.1956 \\
0.1 & 10 & $60^{\circ}$ & 16 & 1.0 & -0.0590 & -0.0539 \\
1.0 & 0.1 & $60^{\circ}$ & 16 & 1.0 & -0.0004 & -0.0004 \\
1.0 & 1.0 & $60^{\circ}$ & 16 & 1.0 & -0.0401 & -0.0426 \\
1.0 & 10 & $60^{\circ}$ & 16 & 1.0 & -0.0636 & -0.0606 \\
10 & 0.1 & $60^{\circ}$ & 16 & 1.0 & 0.0000 & 0.0000 \\
10 & 1.0 & $60^{\circ}$ & 16 & 1.0 & -0.0001 & -0.0001 \\
10 & 10 & $60^{\circ}$ & 16 & 1.0 & -0.0137 & -0.0137 \\
0.1 & 0.1 & $90^{\circ}$ & 32 & 0.5 & -0.0168 & -0.0165 \\
0.1 & 1.0 & $90^{\circ}$ & 16 & 1.0 & -0.2078 & -0.2259 \\
0.1 & 10 & $90^{\circ}$ & 16 & 1.0 & -0.0658 & -0.0623 \\
1.0 & 0.1 & $90^{\circ}$ & 16 & 1.0 & -0.0005 & -0.0005 \\
1.0 & 1.0 & $90^{\circ}$ & 16 & 1.0 & -0.0464 & -0.0492 \\
1.0 & 10 & $90^{\circ}$ & 16 & 1.0 & -0.0715 & -0.0700 \\
10 & 0.1 & $90^{\circ}$ & 16 & 1.0 & 0.0000 & 0.0000 \\
10 & 1.0 & $90^{\circ}$ & 16 & 1.0 & -0.0002 & -0.0002 \\
10 & 10 & $90^{\circ}$ & 16 & 1.0 & -0.0158 & -0.0158 \\
\hline & & & & & &
\end{tabular}




\section{Appendix A. Spherical Bessel function multiplied by appropriate constant numbers}

The explicit expressions of $j_{n}\left(\beta_{m}\right)$ contains $\sin \left[\beta_{m}-(n \pi / 2)\right]$ and $\cos \left[\beta_{m}-(n \pi / 2)\right]$ (e.g., Eq. (10.1.8) of Abramowitz and Stegun (1964)). Because $\beta_{m}=b_{m}-$ $i b_{m}$, where $b_{m} \equiv(m \omega / 2)^{1 / 2}$, the values of $\sin \left[\beta_{m}-(n \pi / 2)\right]$ and $\cos \left[\beta_{m}-(n \pi / 2)\right]$ increase in proportion to $\exp \left(b_{m}\right)$ as $b_{m}$ increases. In order to avoid divergence, we use the following functions instead of $j_{n}\left(\beta_{m}\right)$ and $\beta_{m} j_{n}^{\prime}\left(\beta_{m}\right)$ if $\left|\beta_{m}\right| \gg 1$ :

$$
\begin{aligned}
\hat{j}_{n, m}\left(\beta_{m}\right) & \equiv \beta_{m} j_{n}\left(\beta_{m}\right) / \sin \left[\beta_{m}-(n \pi / 2)\right]=\left[\sum_{\ell=0}^{[n / 2]} \frac{(-i)^{\ell}(n+2 \ell) !}{(2 \ell) !(n-2 \ell) ! 2^{2 \ell}(m \omega)^{\ell}}\right. \\
& \left.-\sum_{\ell=0}^{[(n-1) / 2]} \frac{(-i)^{\ell+1}(n+2 \ell+1) ! \beta_{m}}{(2 \ell+1) !(n-2 \ell-1) ! 2^{2 \ell+1}(m \omega)^{\ell+1}} \cot \left(\beta_{m}-\frac{n \pi}{2}\right)\right],
\end{aligned}
$$

and

$$
\hat{j}_{n, m}^{\prime}\left(\beta_{m}\right) \equiv \beta_{m}^{2} j_{n}^{\prime}\left(\beta_{m}\right) / \sin \left[\beta_{m}-(n \pi / 2)\right]
$$

From Eq. (10.1.20) of Abramowitz and Stegun (1964), we have

$$
\hat{j}_{n, m}^{\prime}\left(\beta_{m}\right)=\frac{\beta_{m} \cot \left[\beta_{m}-(n \pi / 2)\right]}{2 n+1}\left[n \hat{j}_{n-1, m}\left(\beta_{m}\right)+(n+1) \hat{j}_{n+1, m}\left(\beta_{m}\right)\right]
$$

If $\left|\beta_{m}\right| \ll 1, j_{n}\left(\beta_{m}\right)$ and $\beta_{m} j_{n}^{\prime}\left(\beta_{m}\right)$ will be very small, as seen from Eq. (10.1.2) of Abramowitz and Stegun (1964). For a small value of $\left|\beta_{m}\right|$, we use the following functions that are expanded in series of $\beta_{m}^{2}$, instead of $j_{n}\left(\beta_{m}\right)$ and $\beta_{m} j_{n}^{\prime}\left(\beta_{m}\right)$,

$$
\hat{j}_{n, m}\left(\beta_{m}\right) \equiv(2 n+1) ! !\left(\beta_{m}\right)^{-n} j_{n}\left(\beta_{m}\right)=\sum_{\ell=0}^{\infty} \mathcal{J}_{n \ell}\left(\beta_{m}\right)^{2 \ell},
$$


and

$$
\hat{j}_{n, m}^{\prime}\left(\beta_{m}\right) \equiv(2 n+1) ! !\left(\beta_{m}\right)^{1-n} j_{n}^{\prime}\left(\beta_{m}\right)=\sum_{\ell=0}^{\infty}(n+2 \ell) \mathcal{J}_{n \ell}\left(\beta_{m}\right)^{2 \ell},
$$

where

$$
\mathcal{J}_{n \ell} \equiv \frac{(-1)^{\ell}(2 n+1) ! !}{2^{\ell} \ell !(2 n+2 \ell+1) ! !} .
$$

We have performed summation over $0 \leq \ell \leq 1024$ in our numerical calculations.

\section{Acknowledgments}

The authors are thankful to Prof. D. Vokrouhlický and an anonymous reviewer for their helpful comments. S. Wakita is supported by a Grantin-Aid for JSPS Fellows (21-2143) awarded by the Ministry of Education, Culture, Sports, Science and Technology.

\section{References}

Abramowitz, M., Stegun, I.A., 1964. Handbook of Mathematical Functions with Formulas, Graphs, and Mathematical Tables, Dover, New York.

Arfken, G.B., 1970. Mathematical methods for physicists, 2nd ed., Academic Press, New York.

Bottke, W.F.Jr., Vokrouhlický, D., Rubincam, D.P., Nesvorný, D., 2006. The Yarkovsky and YORP effects: Implications for asteroid dynamics, Ann. Rev. Earth Planet. Sci., 34, 157-191.

Breiter, S., Bartczak, P., Czekaj, M., 2010. YORP torques with 1D thermal model, Mon. Not. R. Astron. Soc. 408, 1576-1589. 
Farinella, P., Vokrouhlický, D., Hartmann, W.K., 1998. Meteorite delivery via Yarkovsky orbital drift, Icarus 132, 378-387.

Kadono, T., Arakawa M., Ito, T., Ohtsuki K., 2009. Spin rates of fast-rotating asteroids and fragments in impact disruption, Icarus 200, 694-697.

Lagerros, J.S.V., 1998. Thermal physics of asteroids IV. Thermal infrared beaming, Astron. Astrophys. 332, 1123-1132.

Landau, L.D., Lifshitz, E.M., 1987. Fluid Mechanics, 2nd Ed., trans. Sykes, J.B., Reid, W.H., Butterworth-Heinemann, Oxford.

Opeil, C.P., Consolmagno, G.J., Britt, D.T., 2010. The thermal conductivity of meteorites: New measurements and analysis, Icarus 208, 449-454.

Peterson, C., 1976. A source mechanism for meteorites controlled by the Yarkovsky effect, Icarus 29, 91-111.

Spencer, J.R., 1990. A rough-surface thermophysical model for airless planets, Icarus 83, 27-38.

Spencer, J.R., Lebofsky, L.A., Sykes, M.V., 1989. Systematic biases in radiometric diameter determinations, Icarus 78, 337-354.

Vokrouhlický, D., 1998. Diurnal Yarkovsky effect as a source of mobility of meter-sized asteroidal fragments I. Linear theory, Astron. Astrophys. 335, 1093-1100.

Vokrouhlický, D., Farinella, P., 1999. The Yarkovsky seasonal effect on asteroidal fragments: A nonlinearized theory for spherical bodies, Astron. J. 118, 3049-3060. 
Yomogida, K., Matsui, T., 1983. Physical properties of ordinary chondrites, J. Geophys. Res. 88, 9513-9533. 
Fig. 1

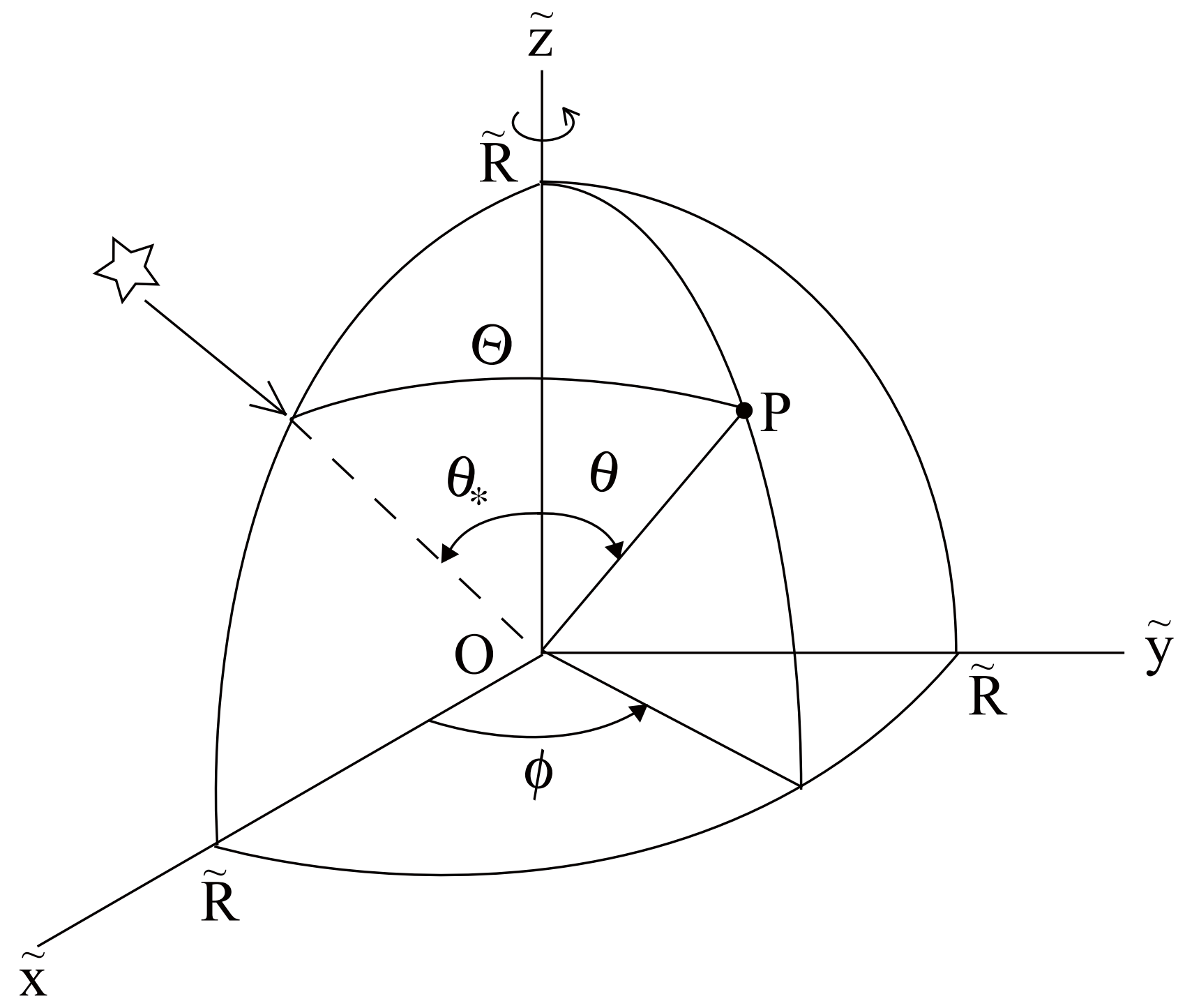


Fig. 2

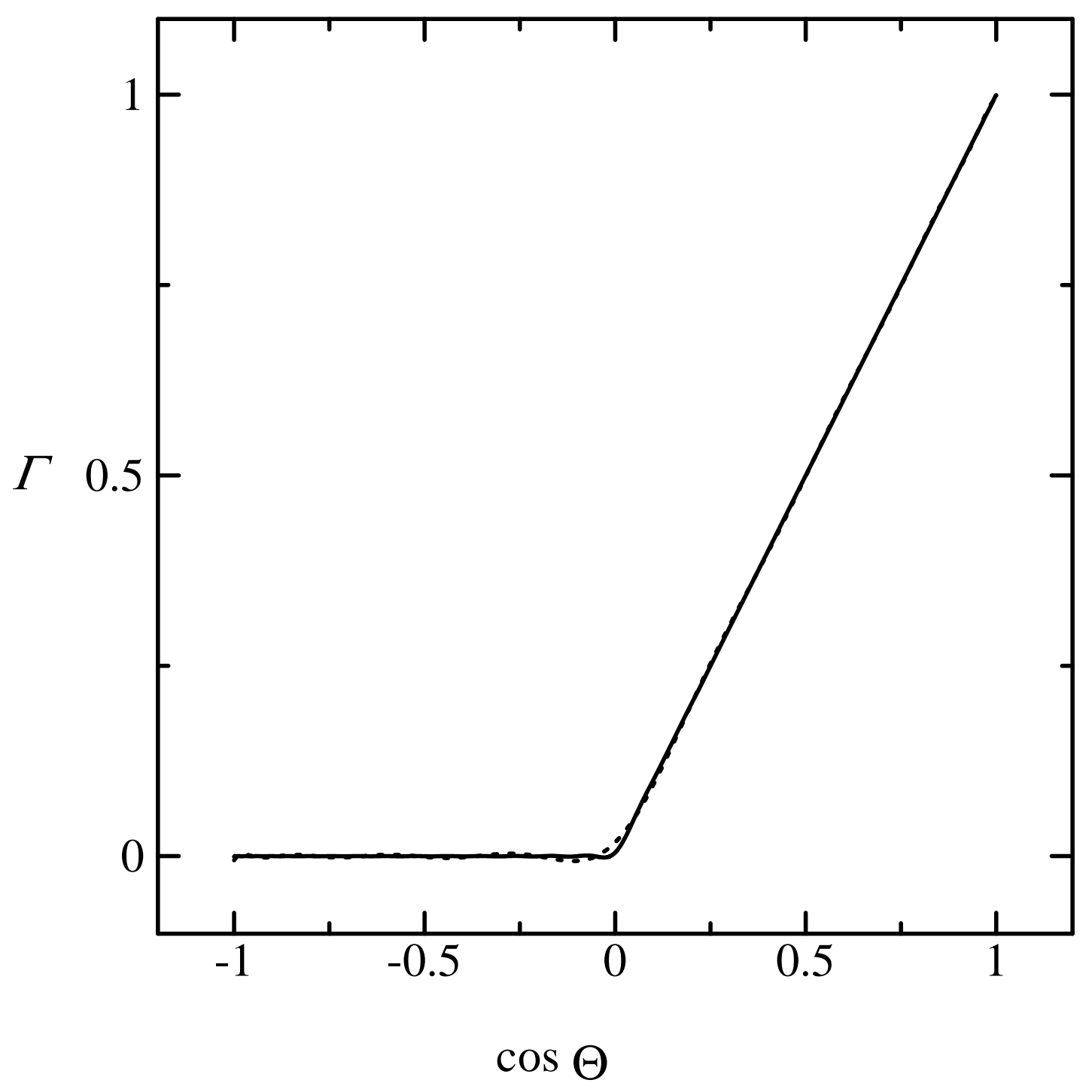


Fig. 3

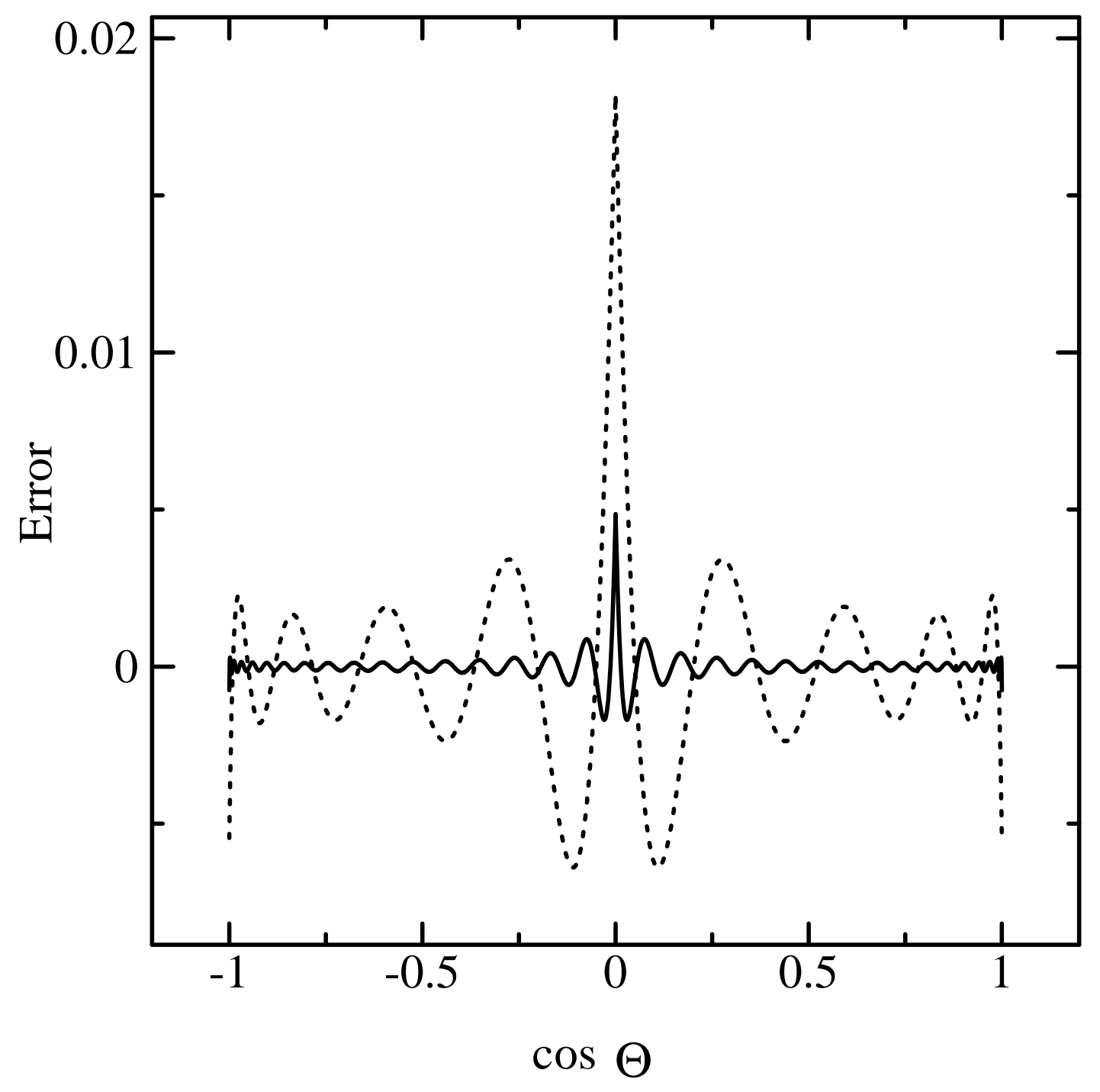


Fig. 4

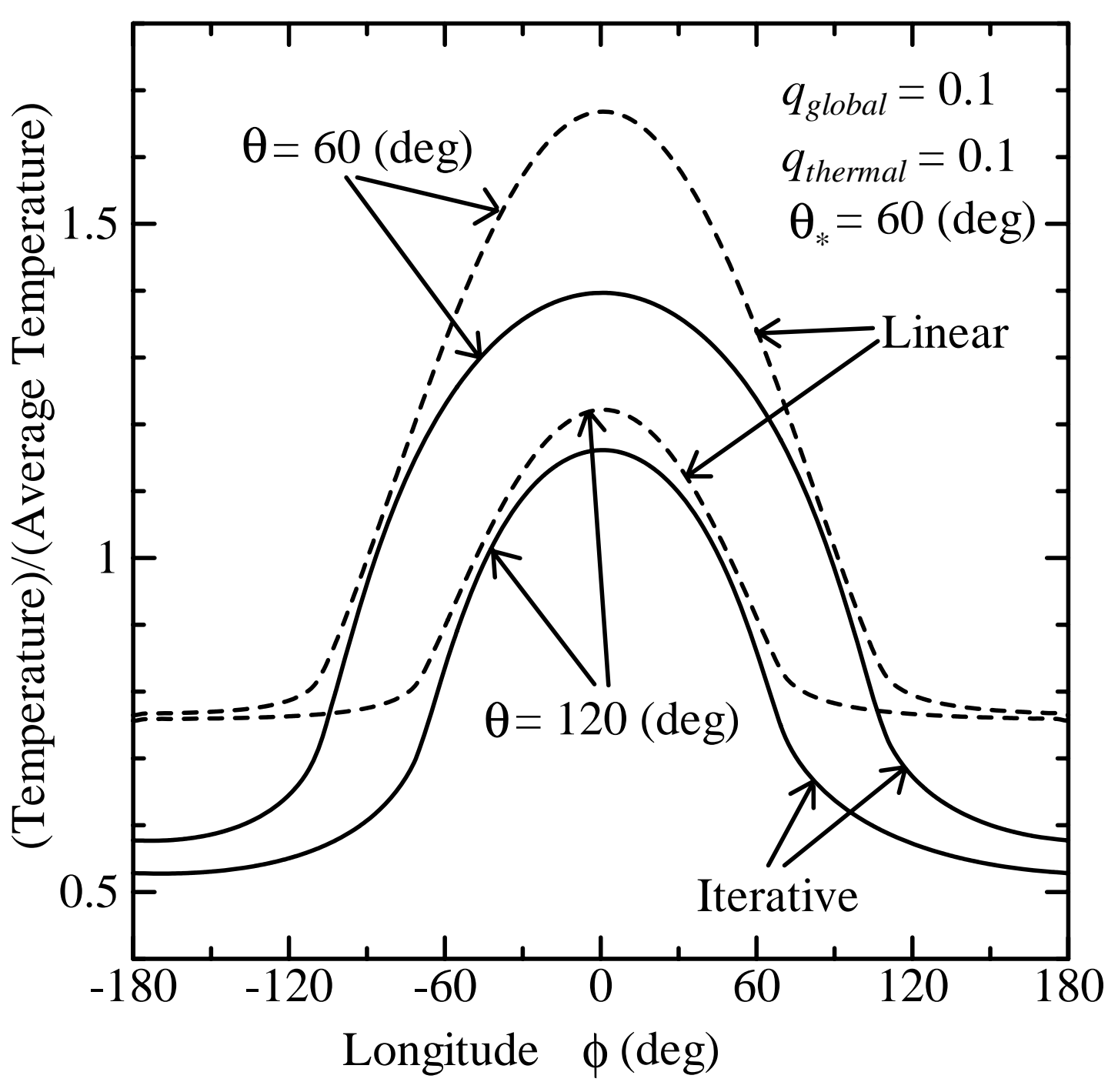


Fig. 5

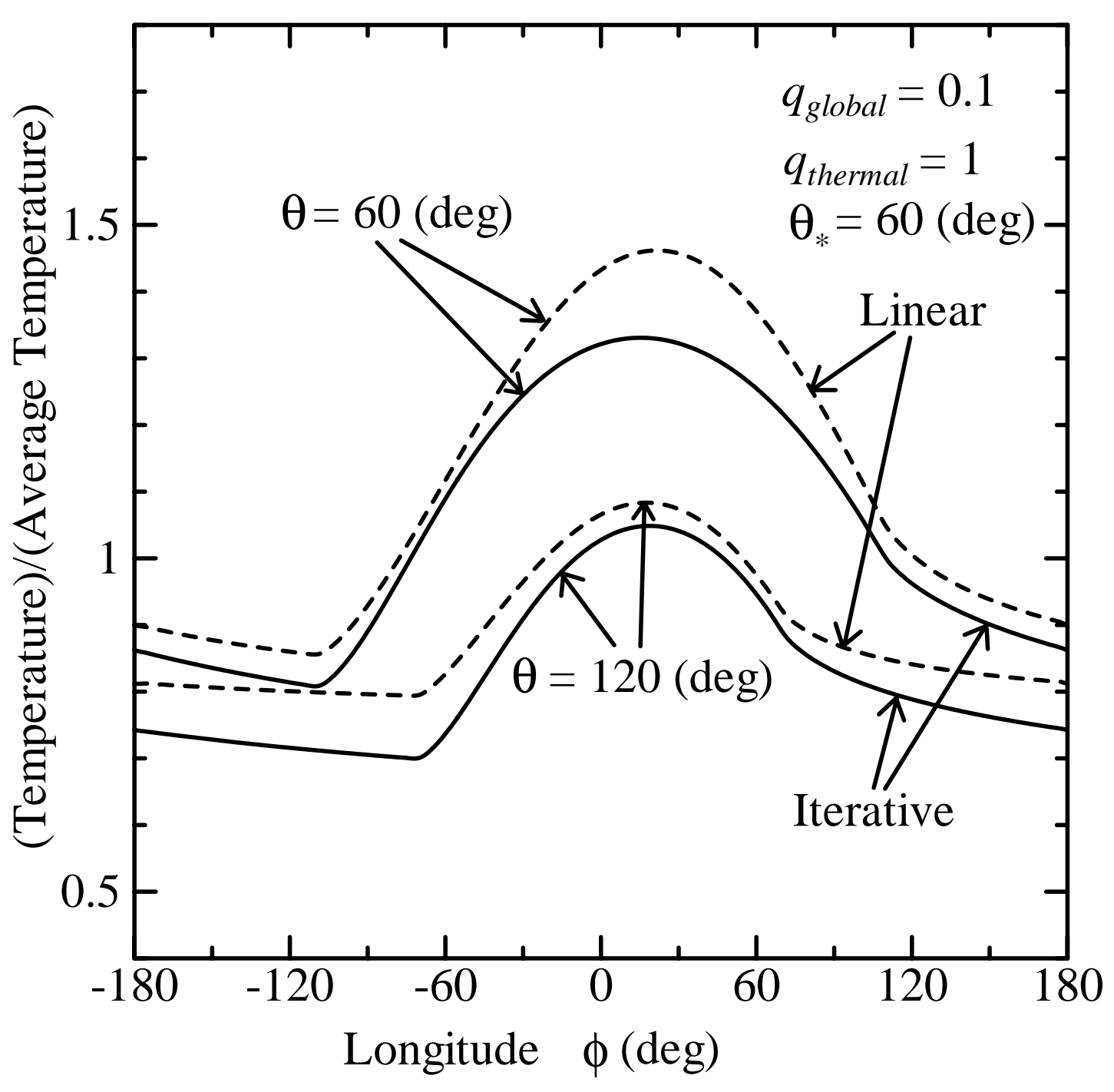


Fig. 6

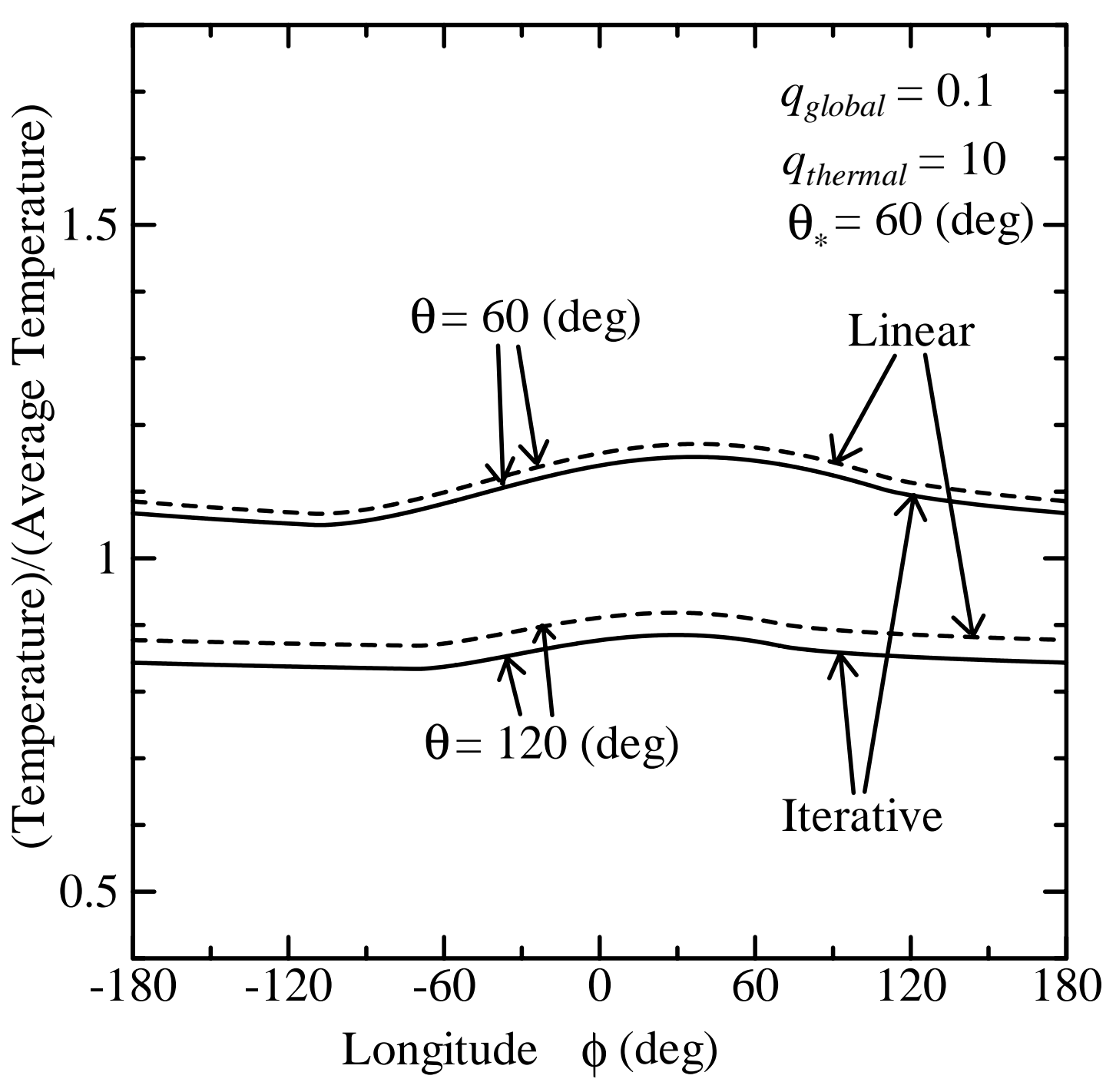


Fig. 7

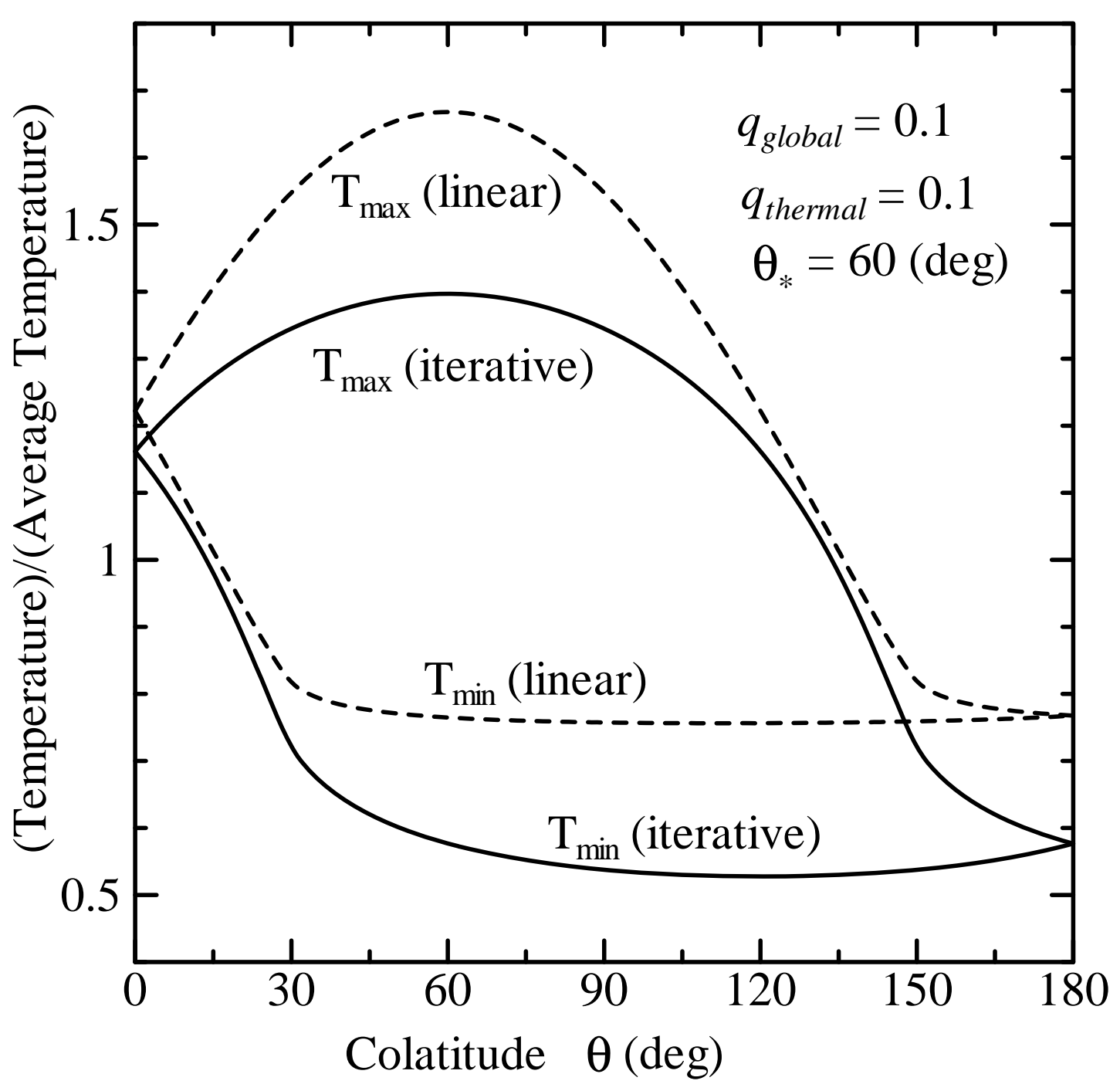


Fig. 8

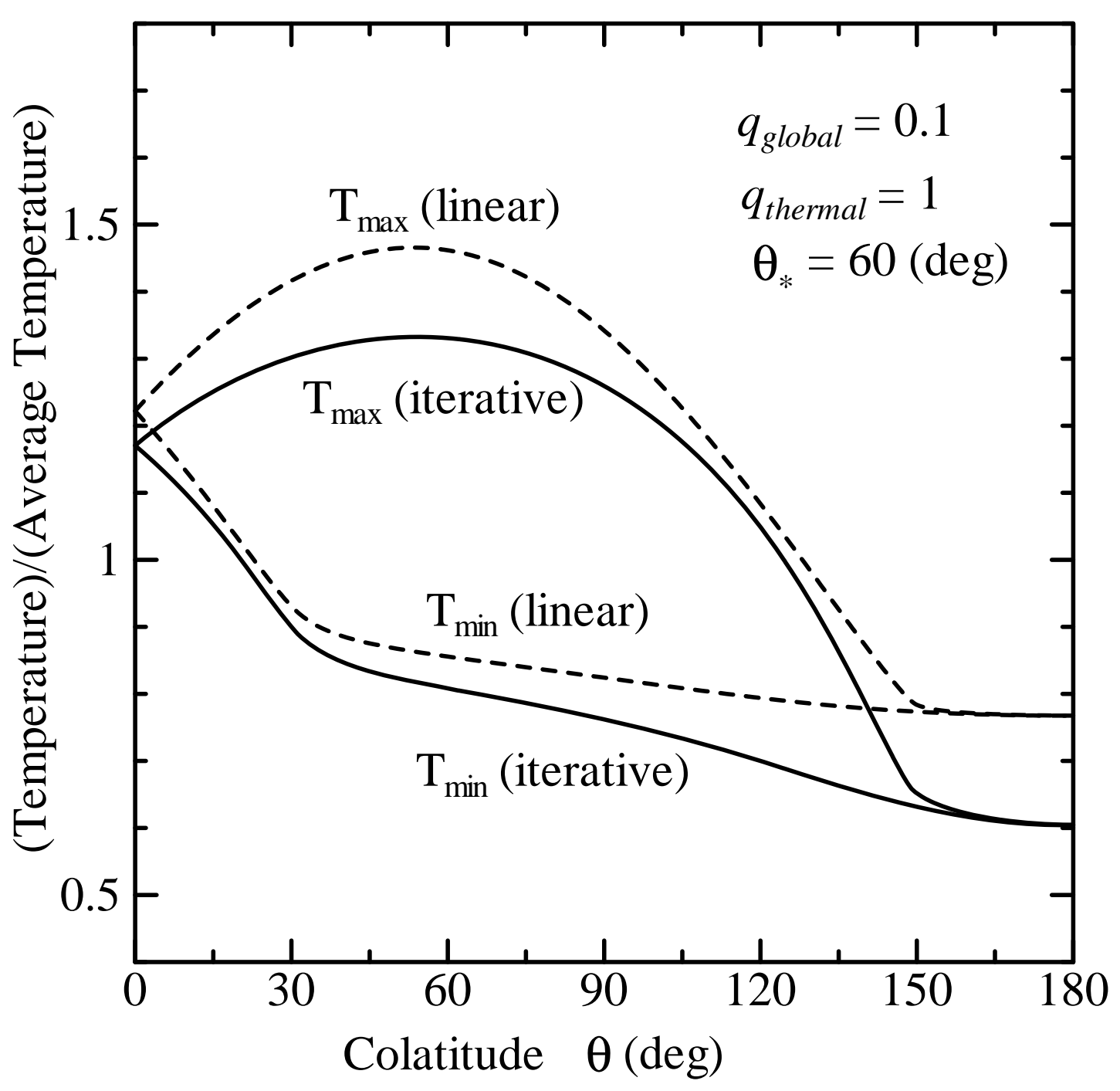


Fig. 9

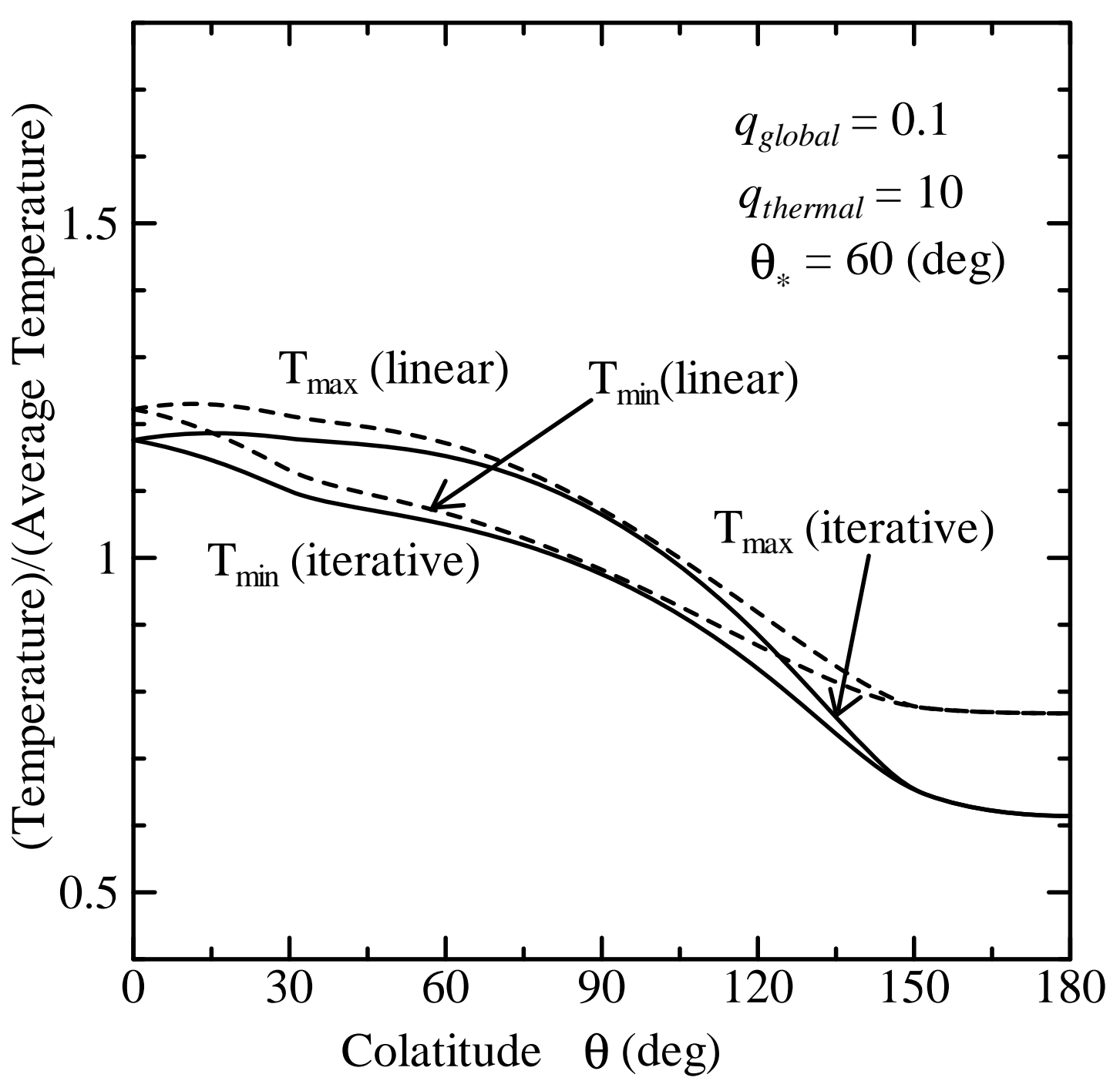


Fig. 10

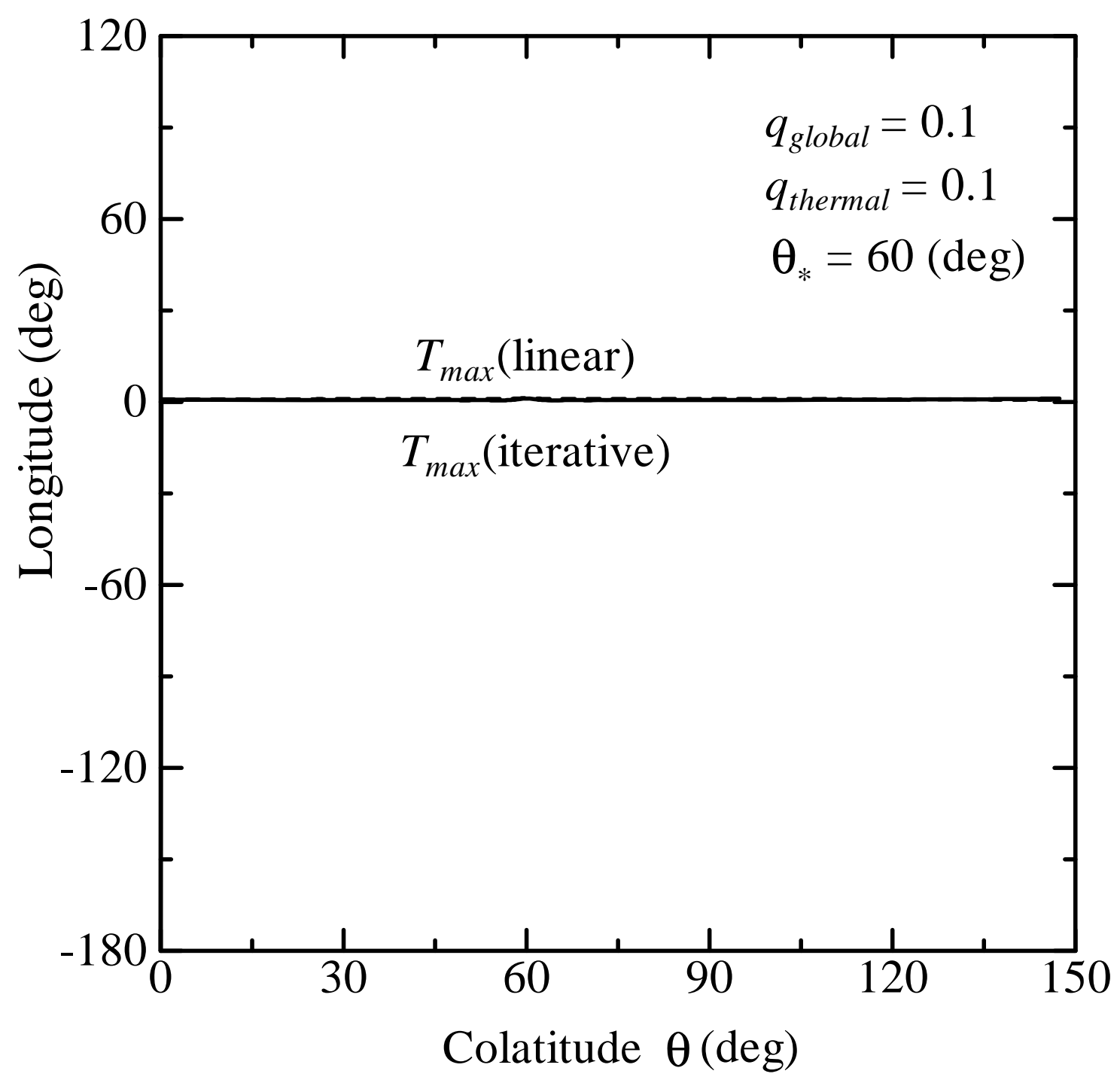


Fig. 11

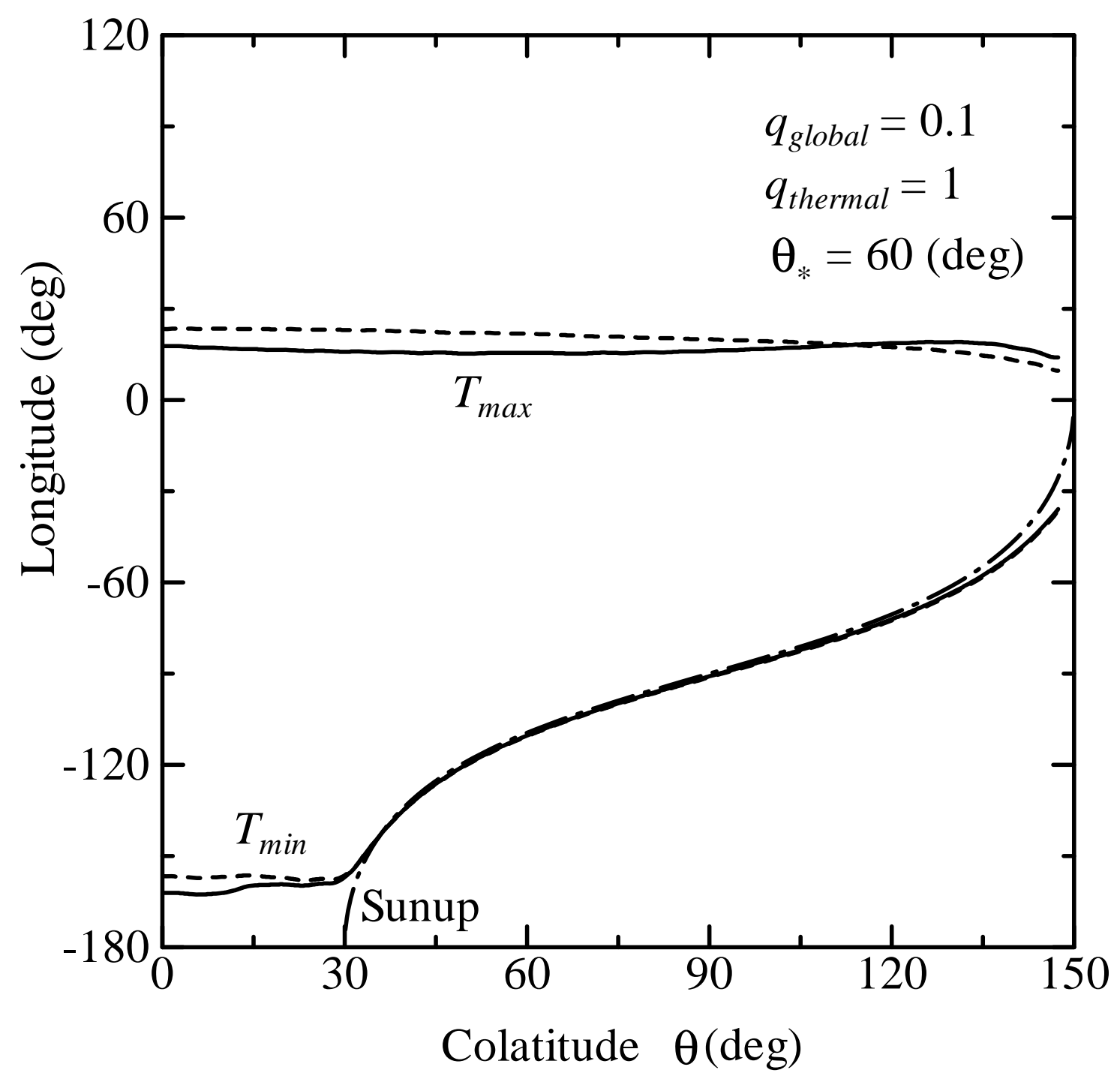


Fig. 12

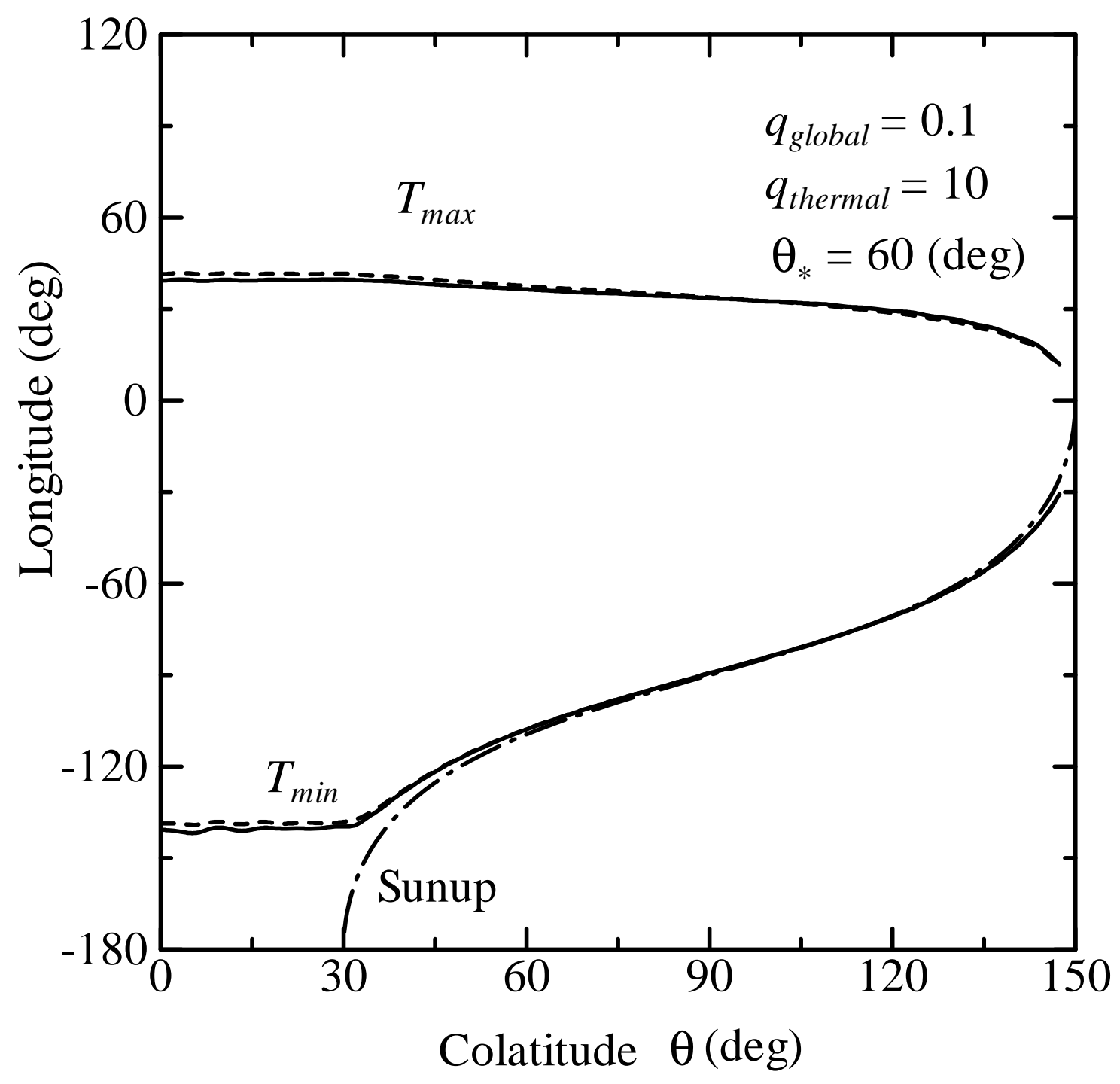


Fig. 13

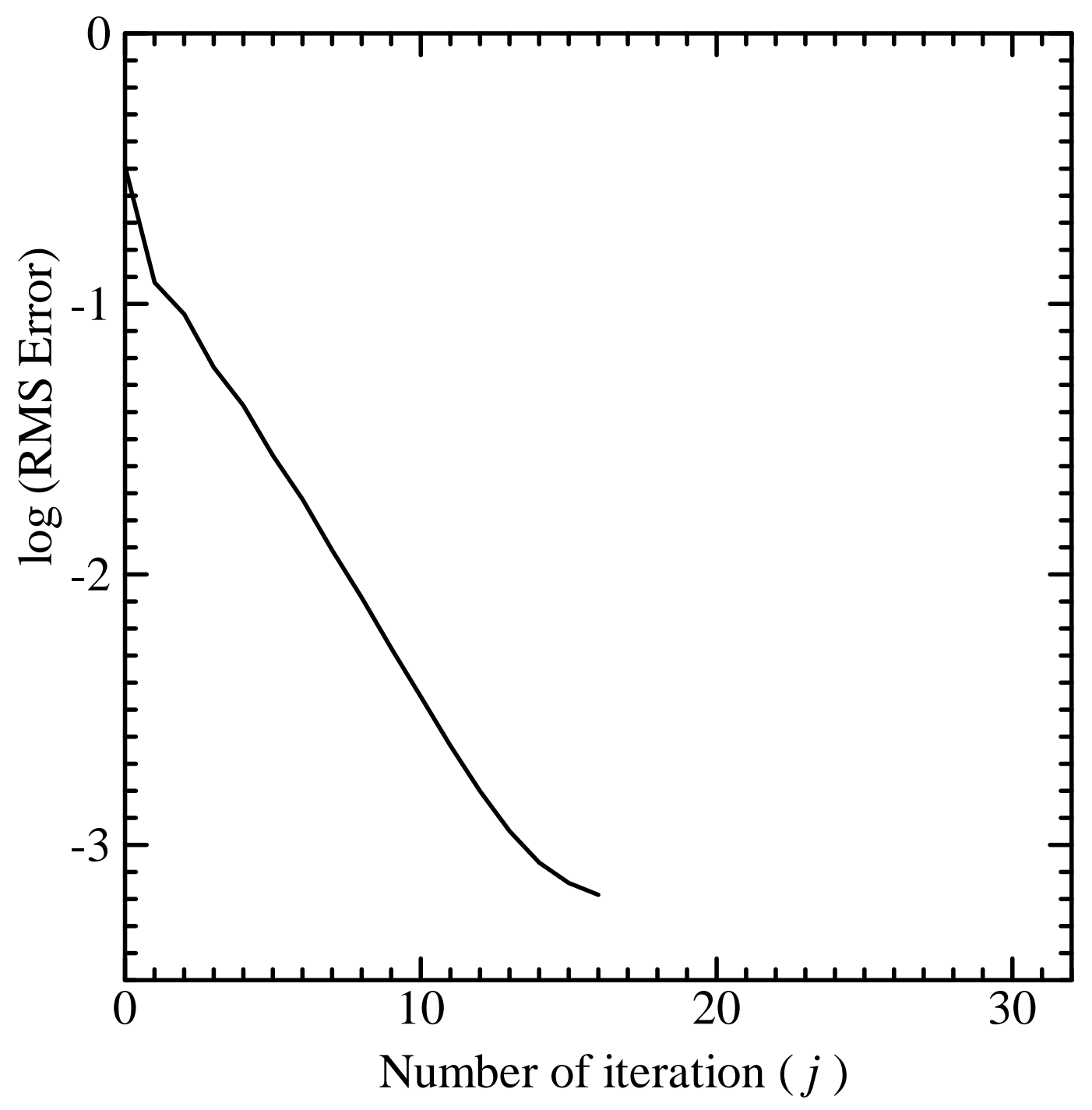


Fig. 14

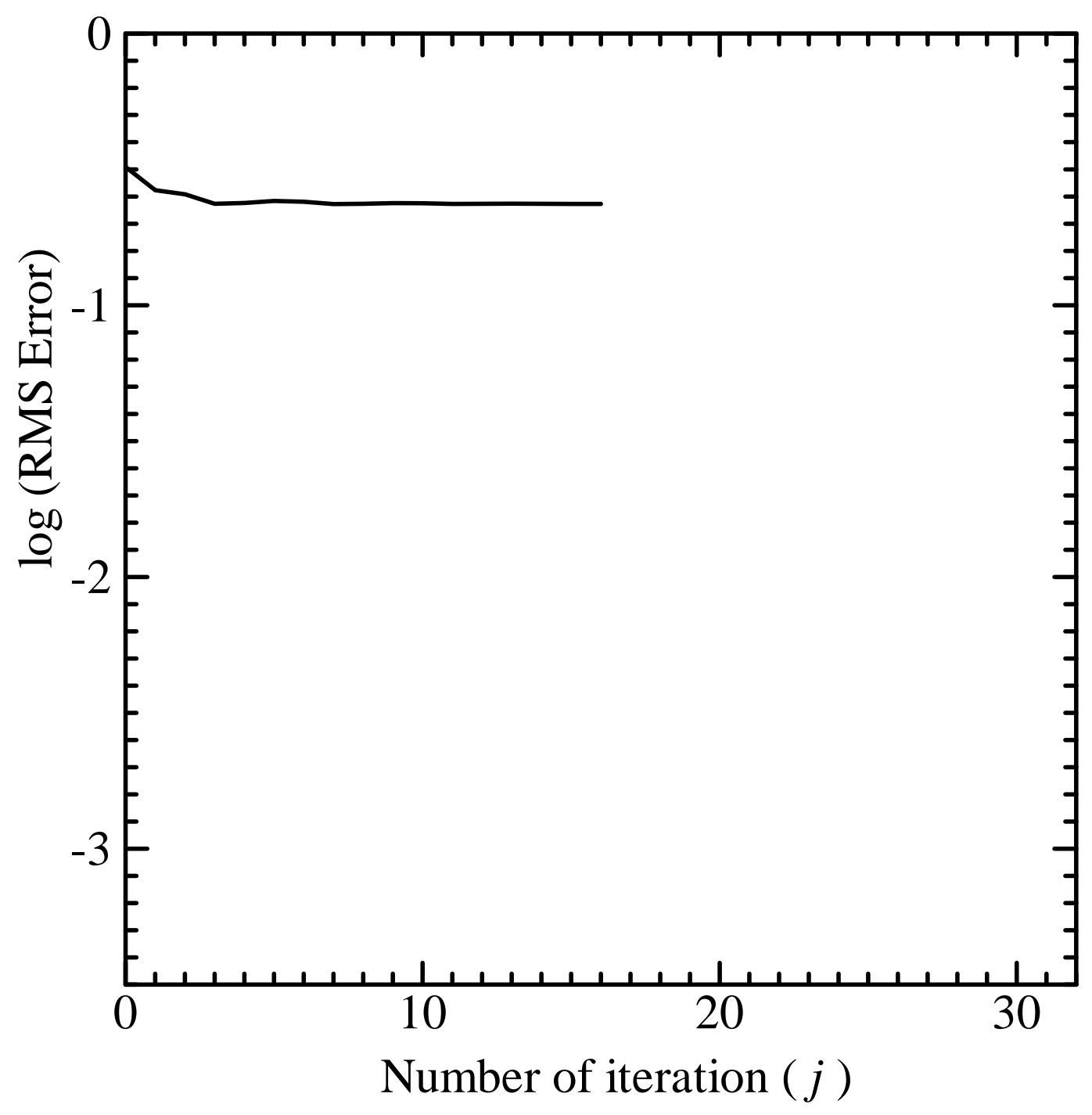


Fig. 15

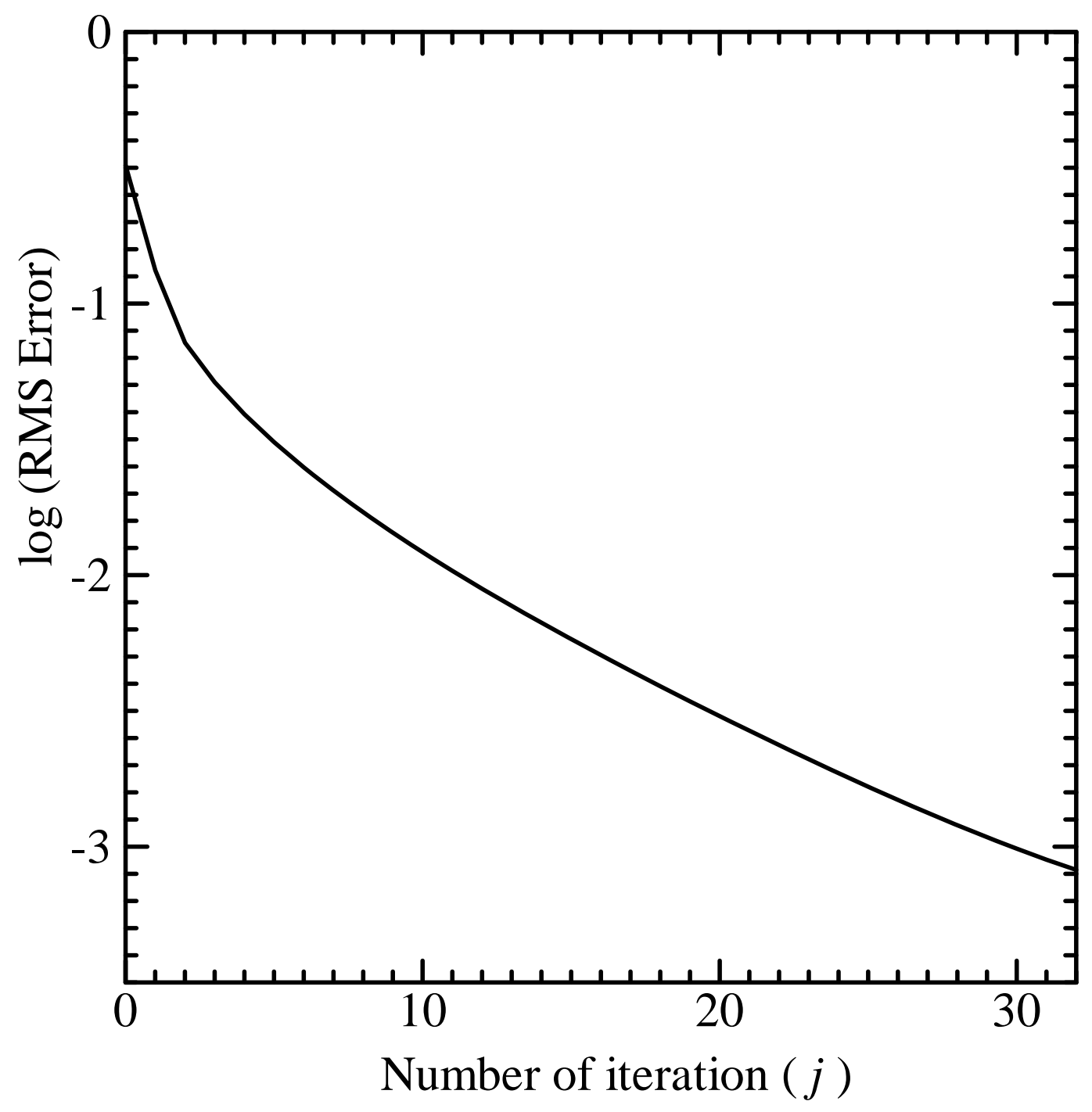


Fig. 16

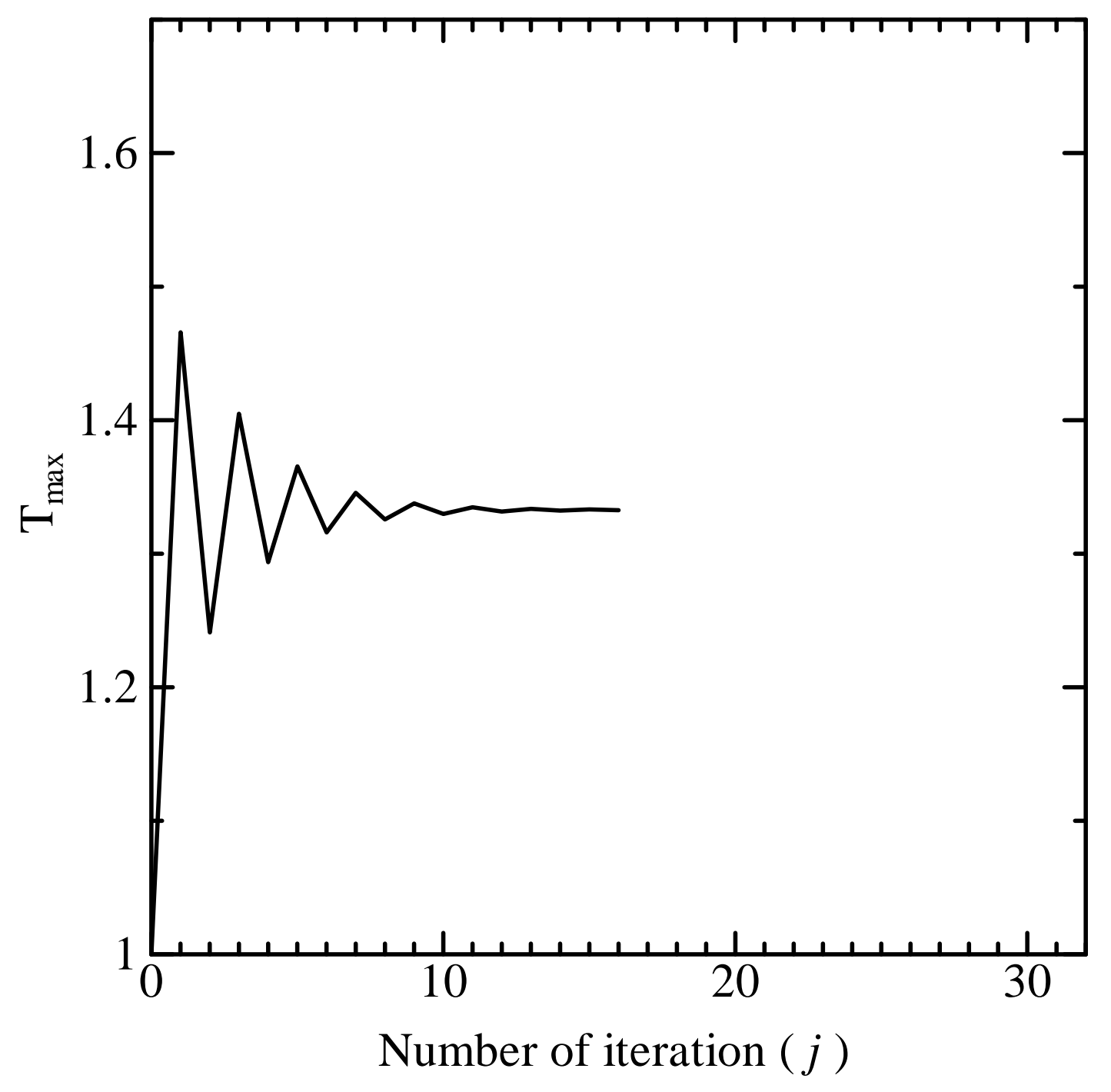


Fig. 17

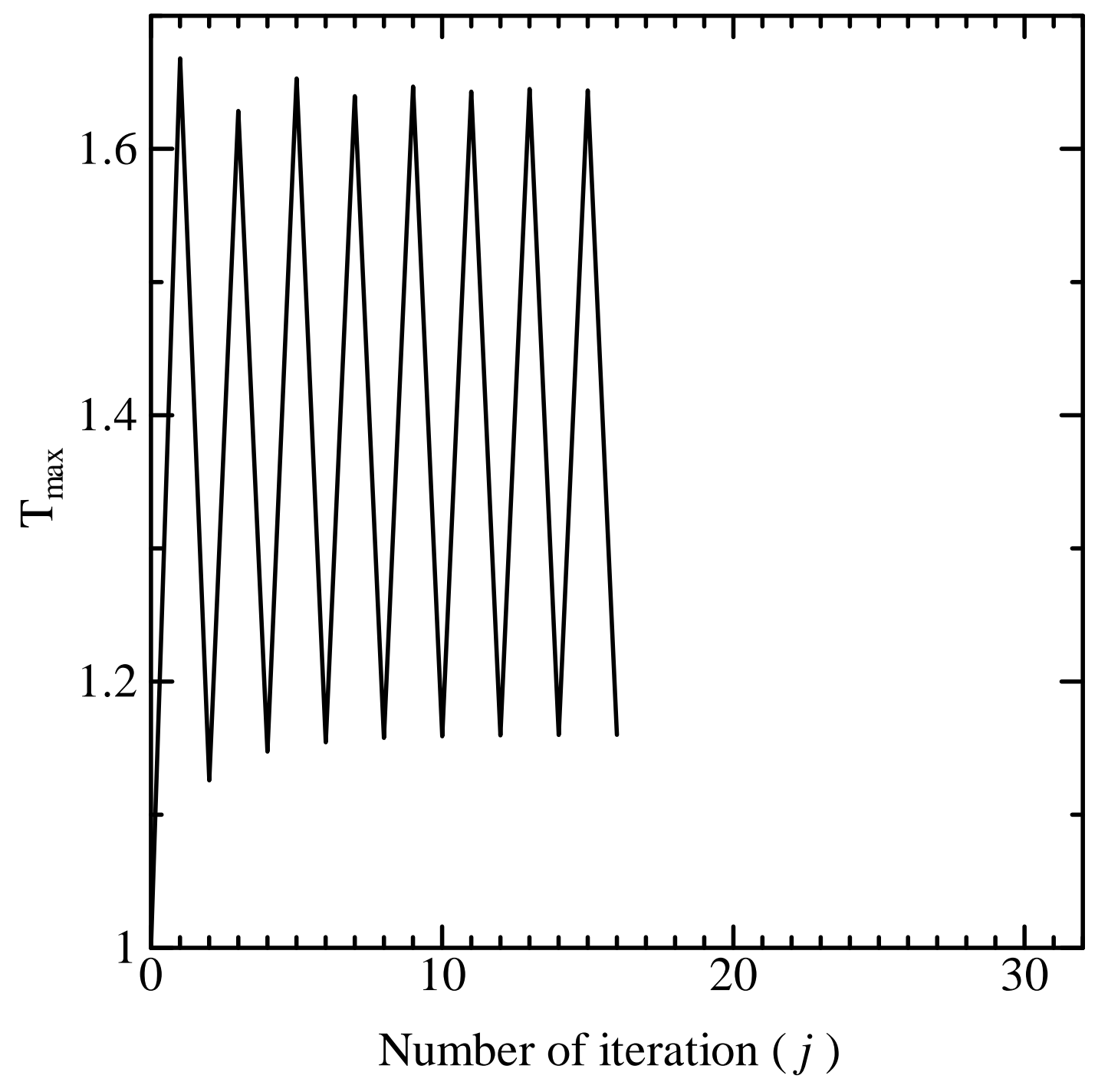


Fig. 18

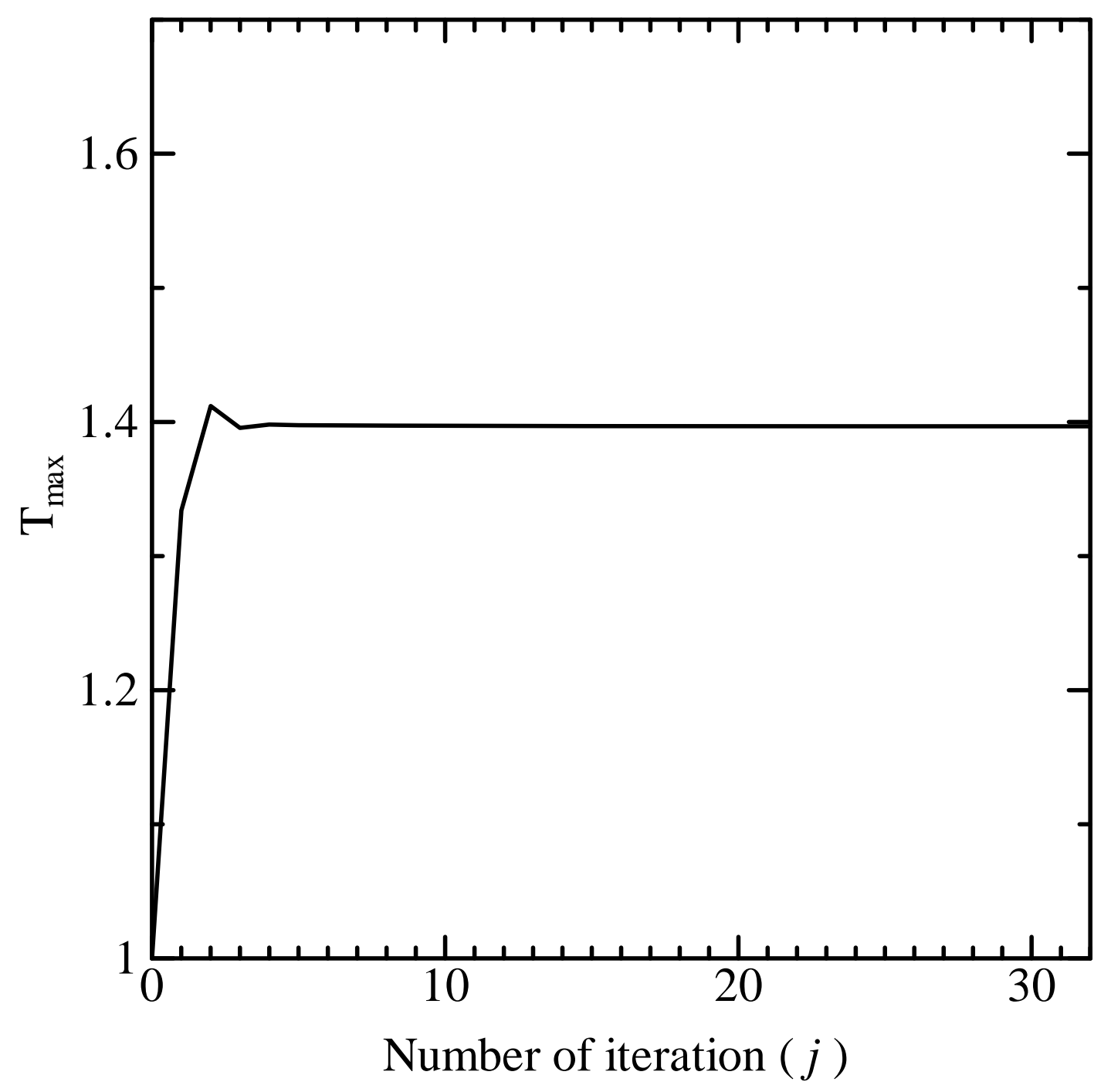

